\title{
A LINEAR SCHEME TO APPROXIMATE NONLINEAR CROSS-DIFFUSION SYSTEMS *
}

\author{
HIDEKI MURAKAWA ${ }^{1}$
}

\begin{abstract}
This paper proposes a linear discrete-time scheme for general nonlinear cross-diffusion systems. The scheme can be regarded as an extension of a linear scheme based on the nonlinear Chernoff formula for the degenerate parabolic equations, which proposed by Berger et al. [RAIRO Anal. Numer. 13 (1979) 297-312]. We analyze stability and convergence of the linear scheme. To this end, we apply the theory of reaction-diffusion system approximation. After discretizing the scheme in space, we obtain a versatile, easy to implement and efficient numerical scheme for the cross-diffusion systems. Numerical experiments are carried out to demonstrate the effectiveness of the proposed scheme.
\end{abstract}

Mathematics Subject Classification. 35K55, 35K57, 65M12, 92D25.

Received October 14, 2010.

Published online July 4, 2011.

\section{INTRODUCTION}

In this paper, we consider discrete-time schemes and numerical schemes to approximate the following nonlinear diffusion problems: Find $\boldsymbol{z}=\left(z_{1}, \ldots, z_{M}\right): \bar{\Omega} \times[0, T) \rightarrow \mathbb{R}^{M}(M \in \mathbb{N})$ such that

$$
\left\{\begin{array}{lll}
\frac{\partial \boldsymbol{z}}{\partial t}=\Delta \boldsymbol{\beta}(\boldsymbol{z})+\boldsymbol{f}(\boldsymbol{z}) & \text { in } & Q:=\Omega \times(0, T), \\
\frac{\partial \boldsymbol{\beta}(\boldsymbol{z})}{\partial \nu}=\mathbf{0} & \text { on } & \partial \Omega \times(0, T), \\
\boldsymbol{z}(\cdot, 0)=\boldsymbol{z}_{0} & \text { in } & \Omega .
\end{array}\right.
$$

Here, $\Omega \subset \mathbb{R}^{d}(d \in \mathbb{N})$ is a bounded domain with smooth boundary $\partial \Omega, T$ is a positive constant, $\boldsymbol{\beta}=$ $\left(\beta_{1}, \ldots, \beta_{M}\right), \boldsymbol{f}=\left(f_{1}, \ldots, f_{M}\right): \mathbb{R}^{M} \rightarrow \mathbb{R}^{M}$ and $\boldsymbol{z}_{0}=\left(z_{01}, \ldots, z_{0 M}\right): \Omega \rightarrow \mathbb{R}^{M}$ are given functions. We note that the diffusivity $\beta_{i}$ of the $i$ th component depends not only on the $i$ th variable but also on the $j$ th $(j \neq i)$ variables in general. This mixture of diffusion terms is called cross-diffusion. Numerous problems of this type have been proposed in the literature, especially in the area of population ecology.

Keywords and phrases. Cross-diffusion systems, nonlinear diffusion, discrete-time schemes, numerical schemes, Reaction-diffusion system approximations.

* This work was supported by Grant-in-Aid for Young Scientists (B)(No. 22740058) from the Ministry of Education, Culture, Sports, Science and Technology of Japan.

${ }^{1}$ Faculty of Mathematics, Kyushu University, 744 Motooka, Nishiku, Fukuoka, 819-0395 Japan. murakawa@math.kyushu-u.ac.jp 
In the case $\beta_{i}(\boldsymbol{z})=a_{i} z_{i}$ for non-negative constants $a_{i}(i=1,2, \ldots, M)$, the problem (1.1) is reduced to a semilinear reaction-diffusion system which are studied in many fields of applications. If the reaction term $\boldsymbol{f}$ is given by

$$
f_{i}(\boldsymbol{z})=\left(g_{i 0}-\sum_{j=1}^{M} g_{i j} z_{j}\right) z_{i}, \quad i=1,2, \ldots, M
$$

for non-negative constants $g_{i j}$, the system is the well-known Lotka-Volterra competition-diffusion system. Here, $z_{i}$ represents the population density of $i$ th species. The constant $a_{i}$ is the diffusion rate, $g_{i 0}$ denotes the intrinsic growth rate, $g_{i i}$ accounts for the intraspecific competition coefficient, and $g_{i j}(i \neq j, j \neq 0)$ are the interspecific competition rates. Qualitative properties of non-negative solutions of this problem have been extensively studied from the mathematical point of view. However, it covers only the case where the diffusive motions of different species are mutually non-interfering. Apart from the point-interactions between species described in (1.2), Kerner [13] considered a motional type of interaction, that recognizes the bias of the motion of predator toward prey and of prey away from predator. He proposed the following cross-diffusion system:

$$
\left\{\begin{array}{l}
\frac{\partial z_{1}}{\partial t}=a_{1} \Delta z_{1}+b_{12} \Delta z_{2}+f_{1}(\boldsymbol{z}) \\
\frac{\partial z_{2}}{\partial t}=a_{2} \Delta z_{2}+b_{21} \Delta z_{1}+f_{2}(\boldsymbol{z})
\end{array}\right.
$$

where $a_{i}(i=1,2)$ are non-negative constants, $b_{12}$ and $b_{21}$ can be either positive or negative. The condition $b_{i j}>0$ denotes the movement of $i$ th species in the direction of lower concentration of $j$ th species, while $b_{i j}<0$ implies that the flux is directed toward increasing population density of the $j$ th species. Gurtin [9] investigated the effect of the cross-diffusion and showed that the effect may give rise to segregation of the two species.

One of the typical problems with cross-diffusion is the following model which was proposed by Shigesada et al. [24] to understand spatial and temporal behaviours of two animal species under the influence of the population pressure due to intra- and interspecific interferences:

$$
\left\{\begin{array}{l}
\frac{\partial z_{1}}{\partial t}=\Delta\left[\left(a_{1}+b_{1} z_{1}+c_{1} z_{2}\right) z_{1}\right]+f_{1}(\boldsymbol{z}) \\
\frac{\partial z_{2}}{\partial t}=\Delta\left[\left(a_{2}+b_{2} z_{2}+c_{2} z_{1}\right) z_{2}\right]+f_{2}(\boldsymbol{z})
\end{array}\right.
$$

where $a_{i}, b_{i}, c_{i}(i=1,2)$ are non-negative constants. The virtual diffusivity of the $i$ th species $a_{i}+b_{i} z_{i}+c_{i} z_{j}$ $(j \neq i)$ is dependent on intra- and interspecific population pressure. The cross-diffusion terms describe tendencies such that the $i$ th species keeps away from high-density areas of the $j$ th species. The spatially segregating coexistence of two competing species occurs by the cross-diffusion effect, which is called cross-diffusion induced instability [15].

There are a number of other exciting cross-diffusion models in population ecology. For instance, Kadota and Kuto [12] investigated the following prey-predator cross-diffusion system.

$$
\left\{\begin{array}{l}
\frac{\partial z_{1}}{\partial t}=\Delta\left[\left(1+b_{1} z_{2}\right) z_{1}\right]+\left(g_{10}-g_{11} z_{1}-g_{12} z_{2}\right) z_{1} \\
\frac{\partial z_{2}}{\partial t}=\Delta\left[\left(a+\frac{1}{1+b_{2} z_{1}}\right) z_{2}\right]+\left(g_{20}+g_{21} z_{1}-g_{22} z_{2}\right) z_{2}
\end{array}\right.
$$

where $a$ and $g_{i j}(i=1,2, j=0,1,2)$ are positive constants, and $b_{1}$ and $b_{2}$ are non-negative constants. In the system, the diffusivity of the prey is the same type of (1.4), but that of the predator is a fractional type which implies the population pressure of the predator weakens in high-density areas of the prey, i.e., the predator migrates towards areas of high concentration of the prey. The same type of nonlinear diffusivity was treated by 
Pang and Wang [23]. They studied a two-predator-one-prey cross-diffusion system in which the prey exercises a defence switching and the predators collaboratively take advantage of the prey's strategy.

Thus, there are a lot of interesting and important cross-diffusion systems. We would like to carry out numerical experiments for various type of nonlinearities. However, there are few results on numerical methods for the cross-diffusion systems. Moreover, numerical methods have been constructed and analyzed for each individual problem separately. To the best of the author's knowledge, there is no effective numerical method for general cross-diffusion systems. The forward difference scheme is versatile and it is very easy to implement. However, the scheme is unstable. The time step size has to be taken to be very small if explicit schemes are used. We need versatile and stable numerical methods for general cross-diffusion systems.

We discuss discrete-time approximations to the problem (1.1). They are simpler than fully-discrete approximations but play a crucial role in developing numerical methods. In this paper, we denote by $\tau=T / N_{T}$ $\left(N_{T} \in \mathbb{N}\right)$ the time step size, and by $\bar{D}_{\tau}$ the backward difference operator, i.e., $\bar{D}_{\tau} Z^{n}=\left(Z^{n}-Z^{n-1}\right) / \tau$ for any given family $\left\{Z^{n}\right\}_{n}$.

We introduce known studies about numerical methods for the Shigesada-Kawasaki-Teramoto model (1.4). Galiano et al. [7] carried out one-dimensional numerical simulations. First they rewrote the diffusion terms and the Neumann boundary conditions as follows:

$$
\begin{gathered}
\frac{\partial z_{i}}{\partial t}=\left(a_{i}+2 b_{i} z_{i}+c_{i} z_{j}\right) \Delta z_{i}+z_{i} \Delta z_{j}+2\left(b_{i} \nabla z_{i}+\nabla z_{j}\right) \cdot \nabla z_{i} \\
\frac{\partial}{\partial \nu}\left[\left(a_{i}+b_{i} z_{i}+c_{i} z_{j}\right) z_{i}\right]=\left(a_{i}+2 b_{i} z_{i}+c_{i} z_{j}\right) \frac{\partial}{\partial \nu} z_{i}+c_{i} z_{i} \frac{\partial}{\partial \nu} z_{j}=0
\end{gathered}
$$

for $(i, j) \in\{(1,2),(2,1)\}$. Then, they chose the following discretization in time.

$$
\begin{gathered}
\bar{D}_{\tau} Z_{i}^{n}=\left(a_{i}+2 b_{i} Z_{i}^{n-1}+c_{i} Z_{j}^{n-1}\right) \Delta Z_{i}^{n}+Z_{i}^{n} \Delta Z_{j}^{n-1}+2\left(b_{i} \nabla Z_{i}^{n-1}+\nabla Z_{j}^{n-1}\right) \cdot \nabla Z_{i}^{n}, \\
\left(a_{i}+2 b_{i} Z_{i}^{n-1}+c_{i} Z_{j}^{n-1}\right) \frac{\partial}{\partial \nu} Z_{i}^{n}+c_{i} Z_{i}^{n} \frac{\partial}{\partial \nu} Z_{j}^{n-1}=0
\end{gathered}
$$

for $n=1,2, \ldots, N_{T}$ with the initial data $Z_{i}^{0}$. This is linear scheme. So, after discretizing in space, the implementation is easy and it's some efficient. However, when the other problem such as (1.5) is considered, another scheme has to be constructed again. Moreover, there is no mathematical results on this scheme. There are theoretical results on numerical methods for the Shigesada-Kawasaki-Teramoto model (1.4). Galiano et al. [8] considered a nonlinear implicit scheme. They proved that convergence in one space dimension. Barrett and Blowey [1] considered a fully discrete finite element approximation with a regularization technique. Their method is also implicit scheme. They showed that convergence in space dimensions $d \leq 3$, and presented numerical experiments in one space dimension. Implicit schemes show better stability and accuracy properties in general. Therefore, their schemes might be efficient. However, for three or four or more components system, the implementation is complicated. Moreover, their analysis can not apply to other cross-diffusion systems. There may be cases where many numerical simulations are carried out by changing not only the coefficients but also the nonlinearity itself. These schemes are not suitable in such cases.

We would like to construct a versatile, easily implemented and efficient numerical method for the general cross-diffusion system (1.1). Investigating numerical methods for single nonlinear diffusion equation $\frac{\partial z}{\partial t}=\Delta \beta(z)$, $z(x, t) \in \mathbb{R}$ may give us a hint. The single equation is still important because this framework is so general as to include the Stefan problems and the porous medium equations. Thereby, a lot of numerical methods have been suggested and analyzed.

Implicit methods of type $\bar{D}_{\tau} Z^{n}=\Delta \beta\left(Z^{n}\right)$ or $\bar{D}_{\tau} \beta^{-1}\left(U^{n}\right)=\Delta U^{n}$ have been extensively studied and they are efficient (see, e.g., $[2,20,22,26]$ and references therein). However, as I mentioned before, the implementation becomes complicated when systems are treated. Implicit methods are good for precise examination of each individual problem. 
Berger et al. [3] suggested the following linear scheme:

$$
\begin{cases}U^{n}-\frac{\tau}{\mu} \Delta U^{n}=\beta\left(Z^{n-1}\right) & \text { in } \quad \Omega, \\ \frac{\partial U^{n}}{\partial \nu}=0 & \text { on } \partial \Omega, \\ Z^{n}:=Z^{n-1}+\mu\left(U^{n}-\beta\left(Z^{n-1}\right)\right) & \text { in } \Omega .\end{cases}
$$

Here, $\mu$ is a positive constant. This scheme is based on nonlinear Chernoff formula arises in nonlinear semigroup theory. This scheme consists of solving a linear elliptic equation and updating $Z$. Many authors investigated this scheme because of its effectiveness (e.g., $[10,11,14,18,19,21,26])$. This scheme can be implemented very easily. Implementation and computational cost are almost same as those for the implicit method for the linear heat equation. When you want to deal with other nonlinearity, all you have to do is to rewrite the function beta. This is the method which we have required. Therefore, we would like to extend this scheme to systems.

The linear scheme (1.7) for the single equation can be extended to that for the nonlinear cross-diffusion system (1.1) immediately. We just replace $U, Z$ and $\beta$ with boldface, that is, vectors; namely,

$$
\begin{cases}\boldsymbol{U}^{n}-\frac{\tau}{\mu} \Delta \boldsymbol{U}^{n}=\boldsymbol{\beta}\left(\boldsymbol{Z}^{n-1}\right)+\frac{\tau}{\mu} \boldsymbol{f}\left(\boldsymbol{Z}^{n-1}\right) & \text { in } \quad \Omega \\ \frac{\partial \boldsymbol{U}^{n}}{\partial \nu}=\mathbf{0} & \text { on } \quad \partial \Omega \\ \boldsymbol{Z}^{n}:=\boldsymbol{Z}^{n-1}+\mu\left(\boldsymbol{U}^{n}-\boldsymbol{\beta}\left(\boldsymbol{Z}^{n-1}\right)\right) & \text { in } \Omega .\end{cases}
$$

This scheme is quite simple. The scheme amounts to solving $M$ linear elliptic equations, followed by explicit algebraic corrections at each time step. The boundary condition is also considerably simpler than that in (1.6). However, the analyses of (1.7) can not be applied to the system (1.8) because they need maximum principle or the primitive function of $\beta$. The maximum principle does not hold for systems in general. And it is difficult to construct the primitive functions of $\boldsymbol{\beta}$ because $\beta_{i}(\boldsymbol{z})$ depends on $z_{j}(j \neq i)$.

Fortunately, we have a clue to solving this problem. We focus on the following semilinear reaction-diffusion system:

$$
\begin{cases}\frac{\partial \boldsymbol{u}}{\partial t}=\frac{1}{\mu} \Delta \boldsymbol{u}-\frac{1}{\varepsilon}(\boldsymbol{u}-\boldsymbol{\beta}(\mu \boldsymbol{u}+\boldsymbol{v}))+\frac{1}{\mu} \boldsymbol{f}(\mu \boldsymbol{u}+\boldsymbol{v}) & \text { in } \quad Q \\ \frac{\partial \boldsymbol{v}}{\partial t}=\frac{\mu}{\varepsilon}(\boldsymbol{u}-\boldsymbol{\beta}(\mu \boldsymbol{u}+\boldsymbol{v})) & \text { in } \quad Q, \\ \frac{\partial \boldsymbol{u}}{\partial \nu}=\mathbf{0} & \text { on } \quad \partial \Omega \times(0, T), \\ \boldsymbol{u}(\cdot, 0)=\boldsymbol{u}_{0}^{\varepsilon}, \quad \boldsymbol{v}(\cdot, 0)=\boldsymbol{v}_{0}^{\varepsilon} & \text { in } \Omega,\end{cases}
$$

where $\mu$ and $\varepsilon$ are positive parameters. This reaction-diffusion system was proposed by the author to avoid the nonlinearity of the diffusion [16]. Let $\left(\boldsymbol{u}^{\varepsilon}, \boldsymbol{v}^{\varepsilon}\right)$ be a weak solution of (1.9). The convergence of $\boldsymbol{z}^{\varepsilon}:=\mu \boldsymbol{u}^{\varepsilon}+\boldsymbol{v}^{\varepsilon}$ to the weak solution $\boldsymbol{z}$ of (1.1) with initial datum $\boldsymbol{z}_{0}=\lim _{\varepsilon \rightarrow 0}\left(\mu \boldsymbol{u}_{0}^{\varepsilon}+\boldsymbol{v}_{0}^{\varepsilon}\right)$ as $\varepsilon$ tends to zero has been shown provided that $\beta_{i}$ depends only on the $i$ th variable. Hence, cross-diffusion systems were not treated. We extend the results to the cross-diffusion system in this paper. The convergence of $\boldsymbol{z}^{\varepsilon}$ to the weak solution of the cross-diffusion systems are shown by imitating a relevant study by the author [17]. In [17], the system (1.1) is approximated by a system of $2 M$ semilinear PDEs while (1.9) consists of $M$ PDEs coupled with $M$ ODEs. The computational costs for ODEs are generally cheaper than those for PDEs. So, the approximation (1.9) is more suitable than the approximation in [17] for constructions of efficient numerical schemes. 
The linear scheme (1.8) can be regarded as a particular time discretization of the reaction-diffusion system (1.9). We consider the following semi-implicit time discretization.

$$
\begin{cases}\bar{D}_{\tau} \boldsymbol{U}^{n}=\frac{1}{\mu} \Delta \boldsymbol{U}^{n}-\frac{1}{\varepsilon}\left(\boldsymbol{U}^{n-1}-\boldsymbol{\beta}\left(\mu \boldsymbol{U}^{n-1}+\boldsymbol{V}^{n-1}\right)\right)+\frac{1}{\mu} \boldsymbol{f}\left(\mu \boldsymbol{U}^{n-1}+\boldsymbol{V}^{n-1}\right) & \text { in } \Omega, \\ \frac{\partial \boldsymbol{U}^{n}}{\partial \nu}=\mathbf{0} & \text { on } \partial \Omega, \\ \bar{D}_{\tau} \boldsymbol{V}^{n}=\frac{\mu}{\varepsilon}\left(\boldsymbol{U}^{n-1}-\boldsymbol{\beta}\left(\mu \boldsymbol{U}^{n-1}+\boldsymbol{V}^{n-1}\right)\right) & \text { in } \Omega .\end{cases}
$$

Put $\boldsymbol{Z}^{n}=\mu \boldsymbol{U}^{n}+\boldsymbol{V}^{n}$. Then, this scheme corresponds to the linear scheme (1.8) if $\varepsilon=\tau$. Indeed, the first equation of (1.10) coincides with the first equation of (1.8) since $\varepsilon=\tau$. The third equation of (1.10) implies

$$
\boldsymbol{V}^{n}=\boldsymbol{Z}^{n-1}-\mu \boldsymbol{\beta}\left(\boldsymbol{Z}^{n-1}\right) .
$$

Add $\mu \boldsymbol{U}^{n}$ both sides to obtain the third relation of (1.8). Thus, (1.8) is regarded as a time discretization of (1.9). Therefore, the idea of proof of the approximation theory can be applied to proof of convergence of the linear scheme (1.8). The aim of this paper is to show stability and convergence of (1.10). As a consequence, we find that $\varepsilon=\tau$ is the best choice while the semi-implicit discretization (1.10) is considered.

The organization of this paper is as follows. In the next section, we state the assumptions and problems precisely. Then, our main result, that is, the convergence of the solution of the discrete-time scheme (1.10) to that of (1.1), is presented. The reaction-diffusion system (1.9) is treated in Section 3. We establish a convergence result of (1.9). Our proof is simple and is based on conventional method: energy estimate and compactness argument. Stability and convergence results of (1.10) are proved in Section 4. In Section 5, numerical simulations are carried out to demonstrate the effectiveness of the linear scheme (1.8).

\section{Formulation AND MAIN RESUlts}

In this section we establish the assumptions on the data, state precisely the problems and present our results.

\subsection{Assumptions}

The general cross-diffusion system are quite difficult to deal with. Even for the problem (1.4), only partial results are available on the existence of solutions (see $[5,6]$ and references therein). We impose the following assumptions:

(H1) $\boldsymbol{\beta}$ is a Lipschitz continuous function satisfying $\boldsymbol{\beta}(\mathbf{0})=\mathbf{0}$.

(H2) There exists a positive constant $a$ such that

$$
\sum_{i=1}^{M} \sum_{j=1}^{M}\left(\beta_{i}\right)_{j}(\boldsymbol{\eta}) \xi_{i} \xi_{j} \geq a|\boldsymbol{\xi}|^{2}
$$

for almost all $\boldsymbol{\eta}, \boldsymbol{\xi} \in \mathbb{R}^{M}$.

Here, $\left(\beta_{i}\right)_{j}$ denotes the derivative of the $i$ th component of $\boldsymbol{\beta}$ with respect to the $j$ th variable.

(H3) $f$ is a Lipschitz continuous function.

Let $L$ be a positive constant satisfying

$$
\left|\left(\beta_{i}\right)_{j}(\boldsymbol{\eta})-a \delta_{i j}\right| \leq L
$$

for almost all $\boldsymbol{\eta} \in \mathbb{R}^{M}$ and all $i, j \in\{1,2, \ldots, M\}$, where $\delta_{i j}$ is the Kronecker delta.

(H4) $\mu$ satisfies

$$
0<\mu<\frac{a}{a^{2}+M^{2} L^{2}} .
$$


(H5) $\boldsymbol{z}_{0} \in L^{2}(\Omega)^{M}$.

(H6) $\tau \leq \varepsilon$ and $\boldsymbol{u}_{0}^{\varepsilon, \tau}, \boldsymbol{v}_{0}^{\varepsilon, \tau} \in H^{1}(\Omega)^{M}$ satisfy

$$
\left\|\mu \boldsymbol{u}_{0}^{\varepsilon, \tau}+\boldsymbol{v}_{0}^{\varepsilon, \tau}\right\|_{L^{2}(\Omega)^{M}}+\sqrt{\tau}\left\|\mu \boldsymbol{u}_{0}^{\varepsilon, \tau}+\boldsymbol{v}_{0}^{\varepsilon, \tau}\right\|_{H^{1}(\Omega)^{M}}+\sqrt{\varepsilon-\tau}\left\|\boldsymbol{v}_{0}^{\varepsilon, \tau}\right\|_{H^{1}(\Omega)^{M}} \leq C
$$

for some positive constant $C$ independent of $\varepsilon$ and $\tau$. Moreover,

$$
\mu \boldsymbol{u}_{0}^{\varepsilon, \tau}+\boldsymbol{v}_{0}^{\varepsilon, \tau} \rightarrow \boldsymbol{z}_{0} \quad \text { weakly in } L^{2}(\Omega)^{M} \text { as } \varepsilon, \tau \rightarrow 0 .
$$

\subsection{Weak formulations}

In this paper, $\langle\cdot, \cdot\rangle$ denotes both the inner product in $L^{2}(\Omega)$ and the duality pairing between $H^{1}(\Omega)^{*}$ and $H^{1}(\Omega)$. The problem (1.1) will be understood in the sense of the following weak form:

Definition 2.1. A function $\boldsymbol{z} \in\left(L^{2}\left(0, T ; H^{1}(\Omega)\right) \cap H^{1}\left(0, T ; H^{1}(\Omega)^{*}\right)\right)^{M}$ is said to be a weak solution of (1.1) if it fulfils

$$
\int_{0}^{T}\left\langle\frac{\partial z_{i}}{\partial t}, \varphi_{i}\right\rangle+\int_{0}^{T}\left\langle\nabla \beta_{i}(\boldsymbol{z}), \nabla \varphi_{i}\right\rangle=\int_{0}^{T}\left\langle f_{i}(\boldsymbol{z}), \varphi_{i}\right\rangle
$$

for all functions $\varphi_{i} \in L^{2}\left(0, T ; H^{1}(\Omega)\right), i=1,2, \ldots, M$, and

$$
\boldsymbol{z}(\cdot, 0)=\boldsymbol{z}_{0} \quad \text { a.e. in } \Omega .
$$

We also introduce a weak form of the time discretization scheme to be studied.

Problem 2.2. We prescribe the initial conditions

$$
\boldsymbol{U}^{0}=\boldsymbol{u}_{0}^{\varepsilon, \tau} \in H^{1}(\Omega)^{M}, \quad \boldsymbol{V}^{0}=\boldsymbol{v}_{0}^{\varepsilon, \tau} \in H^{1}(\Omega)^{M} .
$$

For $i=1,2, \ldots, M$ and $n=1,2, \ldots, N_{T}$, find $U_{i}^{n} \in H^{1}(\Omega)$ and $V_{i}^{n} \in H^{1}(\Omega)$ such that

$$
\left\langle\bar{D}_{\tau} U_{i}^{n}, \varphi\right\rangle+\frac{1}{\mu}\left\langle\nabla U_{i}^{n}, \nabla \varphi\right\rangle=\frac{1}{\mu}\left\langle f_{i}\left(\boldsymbol{Z}^{n-1}\right), \varphi\right\rangle-\frac{1}{\varepsilon}\left\langle U_{i}^{n-1}-\beta_{i}\left(\mu \boldsymbol{U}^{n-1}+\boldsymbol{V}^{n-1}\right), \varphi\right\rangle
$$

for all $\varphi \in H^{1}(\Omega)$, and

$$
\bar{D}_{\tau} V_{i}^{n}=\frac{\mu}{\varepsilon}\left(U_{i}^{n-1}-\beta_{i}\left(\mu \boldsymbol{U}^{n-1}+\boldsymbol{V}^{n-1}\right)\right) \quad \text { a.e. in } \Omega .
$$

Unique solvability of (2.1) besides $U_{i}^{n} \in H^{3}(\Omega)$ are well known (see, e.g., [4]).

\subsection{Main results}

We now state our main results.

Theorem 2.3. Assume that (H1)-(H6) are satisfied. Let $\left\{\boldsymbol{U}^{n}, \boldsymbol{V}^{n}\right\}_{n=0}^{N_{T}}$ be the solution of Problem 2.2. We denote by $\boldsymbol{U}^{(\varepsilon, \tau)}$ and $\boldsymbol{V}^{(\varepsilon, \tau)}$ the functions obtained by piecewise constant interpolation in time of $\left\{\boldsymbol{U}^{n}\right\}$ and $\left\{\boldsymbol{V}^{n}\right\}$, respectively. Then, there exist subsequences $\left\{\boldsymbol{U}^{\left(\varepsilon_{k}, \tau_{k}\right)}\right\},\left\{\boldsymbol{V}^{\left(\varepsilon_{k}, \tau_{k}\right)}\right\}$ of $\left\{\boldsymbol{U}^{(\varepsilon, \tau)}\right\},\left\{\boldsymbol{V}^{(\varepsilon, \tau)}\right\}$ and a weak solution $\boldsymbol{z}$ of (1.1) such that

$$
\begin{array}{ll}
\mu \boldsymbol{U}^{\left(\varepsilon_{k}, \tau_{k}\right)}+\boldsymbol{V}^{\left(\varepsilon_{k}, \tau_{k}\right)} \rightarrow \boldsymbol{z} & \text { strongly in } L^{2}(Q)^{M}, \text { a.e. in } Q \text { and weakly in } L^{2}\left(0, T ; H^{1}(\Omega)\right)^{M}, \\
\boldsymbol{U}^{\left(\varepsilon_{k}, \tau_{k}\right)}-\boldsymbol{\beta}(\boldsymbol{z}) & \text { weakly in } L^{2}\left(0, T ; H^{1}(\Omega)\right)^{M}
\end{array}
$$

as $\varepsilon_{k}$ and $\tau_{k}$ tend to zero. 
The conditions (H1) and (H2) imply that the systems are uniformly parabolic. Therefore, Theorem 2.3 shows that weak solutions of the uniformly parabolic cross-diffusion systems can be approximated by those of the linear scheme (1.8). The cross-diffusion system (1.4) with non-negative bounded solutions is uniformly parabolic if

$$
a_{i}>0,8 b_{1} c_{2}>c_{1}^{2}, 8 b_{2} c_{1}>c_{2}^{2}
$$

The system (1.4) with

$$
a_{i}>0, b_{i}, c_{2} \geq 0, c_{1}=0
$$

is also taken into account. In this case, the system is not always uniformly parabolic, but it is weakly coupled. We can treat more general problems including both cases. We deal with the system (1.1) which possess block triangular diffusion matrices. The diagonal blocks of these matrices have the same structure as the above. We use the following notations: $\mathbb{R}^{M}=\mathbb{R}^{m_{1}} \times \cdots \times \mathbb{R}^{m_{l}}$,

$$
\begin{aligned}
& \boldsymbol{z}=\left(z_{1}, \ldots, z_{n}\right)=\left(z_{11}, \ldots, z_{1 m_{1}}, z_{21}, \ldots, z_{2 m_{2}}, \ldots, z_{l 1}, \ldots, z_{l m_{l}}\right), \\
& \boldsymbol{\beta}=\left(\beta_{1}, \ldots, \beta_{n}\right)=\left(\beta_{11}, \ldots, \beta_{1 m_{1}}, \beta_{21}, \ldots, \beta_{2 m_{2}}, \ldots, \beta_{l 1}, \ldots, \beta_{l m_{l}}\right)
\end{aligned}
$$

and so on. The following condition is imposed on $\boldsymbol{\beta}$ instead of (H2):

$(\mathrm{H} 2)^{\prime}$ For each $i=1, \ldots, l-1$ and $j=1, \ldots, m_{i}$, the function $\beta_{i j}$ is independent of the $r s$ th $(r>i, s=$ $\left.1, \ldots, m_{r}\right)$ variables. Moreover, $\boldsymbol{\beta}$ satisfies

$$
\sum_{j=1}^{m_{i}} \sum_{s=1}^{m_{i}}\left(\beta_{i j}\right)_{i s}(\boldsymbol{\eta}) \xi_{i j} \xi_{i s} \geq a \sum_{j=1}^{m_{i}}\left|\xi_{i j}\right|^{2}
$$

for a positive constant $a$, all $i=1, \ldots, l$ and almost all $\boldsymbol{\eta}, \boldsymbol{\xi} \in \mathbb{R}^{M}$.

Combining the proof of Theorem 2.3 with the idea of the proof in [17], we obtain the following result.

Corollary 2.4. Assume that (H1), (H2)' and (H3)-(H6) are satisfied. Let $\left\{\boldsymbol{U}^{n}, \boldsymbol{V}^{n}\right\}_{n=0}^{N_{T}}$ be the solution of Problem 2.2. We denote by $\boldsymbol{U}^{(\varepsilon, \tau)}$ and $\boldsymbol{V}^{(\varepsilon, \tau)}$ the functions obtained by piecewise constant interpolation in time of $\left\{\boldsymbol{U}^{n}\right\}$ and $\left\{\boldsymbol{V}^{n}\right\}$, respectively. Then, there exist subsequences $\left\{\boldsymbol{U}^{\left(\varepsilon_{k}, \tau_{k}\right)}\right\},\left\{\boldsymbol{V}^{\left(\varepsilon_{k}, \tau_{k}\right)}\right\}$ of $\left\{\boldsymbol{U}^{(\varepsilon, \tau)}\right\},\left\{\boldsymbol{V}^{(\varepsilon, \tau)}\right\}$ and a weak solution $\boldsymbol{z}$ of (1.1) such that

$$
\begin{array}{ll}
\mu \boldsymbol{U}^{\left(\varepsilon_{k}, \tau_{k}\right)}+\boldsymbol{V}^{\left(\varepsilon_{k}, \tau_{k}\right)} \rightarrow \boldsymbol{z} & \text { strongly in } L^{2}(Q)^{M}, \text { a.e. in } Q \text { and weakly in } L^{2}\left(0, T ; H^{1}(\Omega)\right)^{M}, \\
\boldsymbol{U}^{\left(\varepsilon_{k}, \tau_{k}\right)} \rightarrow \boldsymbol{\beta}(\boldsymbol{z}) & \text { weakly in } L^{2}\left(0, T ; H^{1}(\Omega)\right)^{M}
\end{array}
$$

as $\varepsilon_{k}$ and $\tau_{k}$ tend to zero.

\section{A REACTION-DIFFUSION SYSTEM APPROXIMATION}

In this section, we analyze the reaction-diffusion system (1.9).

\subsection{Formulation and results}

The following condition is imposed on the initial data instead of (H6):

$(\mathrm{H} 6)^{\prime} \boldsymbol{u}_{0}^{\varepsilon} \in L^{2}(\Omega)^{M}$ and $\boldsymbol{v}_{0}^{\varepsilon} \in H^{1}(\Omega)^{M}$ satisfy

$$
\left\|\mu \boldsymbol{u}_{0}^{\varepsilon}+\boldsymbol{v}_{0}^{\varepsilon}\right\|_{L^{2}(\Omega)^{M}}+\sqrt{\varepsilon}\left\|\boldsymbol{v}_{0}^{\varepsilon}\right\|_{H^{1}(\Omega)^{M}} \leq C
$$

for some positive constant $C$ independent of $\varepsilon$, and

$$
\mu \boldsymbol{u}_{0}^{\varepsilon}+\boldsymbol{v}_{0}^{\varepsilon} \rightarrow \boldsymbol{z}_{0} \quad \text { weakly in } L^{2}(\Omega)^{M} .
$$

We introduce a weak formulation of (1.9). 
Definition 3.1. We call $(\boldsymbol{u}, \boldsymbol{v}) \in\left(L^{2}\left(0, T ; H^{1}(\Omega)\right) \cap H^{1}\left(0, T ; H^{1}(\Omega)^{*}\right)\right)^{M} \times H^{1}(Q)^{M}$ a weak solution of (1.9) if it satisfies

$$
\int_{0}^{T}\left\langle\frac{\partial u_{i}}{\partial t}, \varphi_{i}\right\rangle+\frac{1}{\mu} \int_{0}^{T}\left\langle\nabla u_{i}, \nabla \varphi_{i}\right\rangle=\frac{1}{\mu} \int_{0}^{T}\left\langle f_{i}(\mu \boldsymbol{u}+\boldsymbol{v}), \varphi_{i}\right\rangle-\frac{1}{\varepsilon} \int_{0}^{T}\left\langle u_{i}-\beta_{i}(\mu \boldsymbol{u}+\boldsymbol{v}), \varphi_{i}\right\rangle
$$

for all functions $\varphi_{i} \in L^{2}\left(0, T ; H^{1}(\Omega)\right)$ and

$$
\frac{\partial v_{i}}{\partial t}=\frac{\mu}{\varepsilon}\left(u_{i}-\beta_{i}(\mu \boldsymbol{u}+\boldsymbol{v})\right) \quad \text { a.e. in } Q .
$$

for $i=1,2, \ldots, M$, and

$$
\boldsymbol{u}(\cdot, 0)=\boldsymbol{u}_{0}^{\varepsilon}, \quad \boldsymbol{v}(\cdot, 0)=\boldsymbol{v}_{0}^{\varepsilon} \quad \text { a.e. in } \Omega .
$$

The unique existence of the weak solution of (1.9) can be established by means of the conventional method (see also Rem. 4.2 for existence). For any $\varepsilon>0$ the problem (1.9) has one and only one weak solution $\left(\boldsymbol{u}^{\varepsilon}, \boldsymbol{v}^{\varepsilon}\right)$ such that

$$
\begin{aligned}
& \boldsymbol{u}^{\varepsilon} \in\left(L^{\infty}\left(0, T ; L^{2}(\Omega)\right) \cap L^{2}\left(0, T ; H^{1}(\Omega)\right) \cap H^{1}\left(0, T ; H^{1}(\Omega)^{*}\right)\right)^{M}, \\
& \boldsymbol{v}^{\varepsilon} \in\left(L^{\infty}\left(0, T ; H^{1}(\Omega)\right) \cap H^{1}(Q)\right)^{M} .
\end{aligned}
$$

We have prepared sufficiently to state our results.

Theorem 3.2. Assume that (H1)-(H5) and $(\mathrm{H} 6)^{\prime}$ hold. Let $\left(\boldsymbol{u}^{\varepsilon}, \boldsymbol{v}^{\varepsilon}\right)$ be the weak solution of (1.9). Then, there exist a weak solution $\boldsymbol{z} \in\left(L^{\infty}\left(0, T ; L^{2}(\Omega)\right) \cap L^{2}\left(0, T ; H^{1}(\Omega)\right) \cap H^{1}\left(0, T ; H^{1}(\Omega)^{*}\right)\right)^{M}$ of (1.1) and subsequences $\left\{\boldsymbol{u}^{\varepsilon_{k}}\right\},\left\{\boldsymbol{v}^{\varepsilon_{k}}\right\}$ of $\left\{\boldsymbol{u}^{\varepsilon}\right\},\left\{\boldsymbol{v}^{\varepsilon}\right\}$ such that

$$
\begin{array}{ll}
\mu \boldsymbol{u}^{\varepsilon_{k}}+\boldsymbol{v}^{\varepsilon_{k}} \rightarrow \boldsymbol{z} & \text { strongly in } L^{2}(Q)^{M}, \text { a.e. in } Q, \\
\boldsymbol{u}^{\varepsilon_{k}} \rightarrow \boldsymbol{\beta}(\boldsymbol{z}) & \text { and weakly in } L^{2}\left(0, T ; H^{1}(\Omega)\right)^{M} \\
& \text { weakly in } L^{2}\left(0, T ; H^{1}(\Omega)\right)^{M}
\end{array}
$$

as $\varepsilon_{k}$ tends to zero.

Our results show that the weak solutions of the cross-diffusion systems can be approximated by those of the reaction-diffusion system (1.9).

We can deal with the general problem so that $(\mathrm{H} 2)^{\prime}$ is satisfied instead of (H2).

Corollary 3.3. Assume that (H1), (H2)', (H3)-(H5) and $(\mathrm{H} 6)^{\prime}$ hold. Let $\left(\boldsymbol{u}^{\varepsilon}, \boldsymbol{v}^{\varepsilon}\right)$ be the weak solution of (1.9). Then, there exist a weak solution $\boldsymbol{z} \in\left(L^{\infty}\left(0, T ; L^{2}(\Omega)\right) \cap L^{2}\left(0, T ; H^{1}(\Omega)\right) \cap H^{1}\left(0, T ; H^{1}(\Omega)^{*}\right)\right)^{M}$ of $(1.1)$ and subsequences $\left\{\boldsymbol{u}^{\varepsilon_{k}}\right\},\left\{\boldsymbol{v}^{\varepsilon_{k}}\right\}$ of $\left\{\boldsymbol{u}^{\varepsilon}\right\},\left\{\boldsymbol{v}^{\varepsilon}\right\}$ such that

$$
\begin{array}{ll}
\mu \boldsymbol{u}^{\varepsilon_{k}}+\boldsymbol{v}^{\varepsilon_{k}} \rightarrow \boldsymbol{z} & \text { strongly in } L^{2}(Q)^{M}, \text { a.e. in } Q, \\
\boldsymbol{u}^{\varepsilon_{k}} \rightarrow \boldsymbol{\beta}(\boldsymbol{z}) & \text { and weakly in } L^{2}\left(0, T ; H^{1}(\Omega)\right)^{M} \\
& \text { weakly in } L^{2}\left(0, T ; H^{1}(\Omega)\right)^{M}
\end{array}
$$

as $\varepsilon_{k}$ tends to zero.

\subsection{A priori estimates}

A priori estimates are presented for the weak solution $\left(\boldsymbol{u}^{\varepsilon}, \boldsymbol{v}^{\varepsilon}\right)$ of the system (1.9) and a function $\boldsymbol{z}^{\varepsilon}=$ $\mu \boldsymbol{u}^{\varepsilon}+\boldsymbol{v}^{\varepsilon}$. In this section, $C$ denotes a generic positive constant independent of $\varepsilon$. 
Lemma 3.4. Assume that $(\mathrm{H} 1)-(\mathrm{H} 4)$ and $(\mathrm{H} 6)^{\prime}$ are satisfied. Then there exists a positive constant $C$ independent of $\varepsilon$ such that

$$
\begin{array}{r}
\left\|\boldsymbol{z}^{\varepsilon}\right\|_{\left.\left(L^{\infty}\left(0, T ; L^{2}(\Omega)\right) \cap L^{2}\left(0, T ; H^{1}(\Omega)\right) \cap H^{1}\left(0, T ; H^{1}(\Omega)\right)^{*}\right)\right)^{M}}+\left\|\boldsymbol{u}^{\varepsilon}\right\|_{L^{2}\left(0, T ; H^{1}(\Omega)\right)^{M}} \\
+\left\|\boldsymbol{v}^{\varepsilon}\right\|_{L^{2}\left(0, T ; H^{1}(\Omega)\right)^{M}}+\sqrt{\varepsilon}\left\|\boldsymbol{v}^{\varepsilon}\right\|_{L^{\infty}\left(0, T ; H^{1}(\Omega)\right)^{M}} \leq C .
\end{array}
$$

Proof. From Definition 3.1, the functions $\boldsymbol{z}^{\varepsilon}$ and $\boldsymbol{u}^{\varepsilon}$ satisfy

$$
\int_{0}^{T}\left\langle\frac{\partial z_{i}^{\varepsilon}}{\partial t}, \varphi_{i}\right\rangle+\int_{0}^{T}\left\langle\nabla u_{i}^{\varepsilon}, \nabla \varphi_{i}\right\rangle=\int_{0}^{T}\left\langle f_{i}\left(\boldsymbol{z}^{\varepsilon}\right), \varphi_{i}\right\rangle
$$

for all functions $\varphi_{i} \in L^{2}\left(0, T ; H^{1}(\Omega)\right)$ and $i=1, \ldots, M$. For an arbitrary point $t_{0} \in(0, T)$, take

$$
\varphi_{i}(\cdot, t)= \begin{cases}z_{i}^{\varepsilon}(\cdot, t) & \text { for } 0<t<t_{0} \\ 0 & \text { otherwise }\end{cases}
$$

to obtain

$$
\frac{1}{2}\left\|z_{i}^{\varepsilon}\left(t_{0}\right)\right\|_{L^{2}(\Omega)}^{2}-\frac{1}{2}\left\|z_{0 i}^{\varepsilon}\right\|_{L^{2}(\Omega)}^{2}+\int_{0}^{t_{0}}\left\langle\nabla u_{i}^{\varepsilon}, \nabla z_{i}^{\varepsilon}\right\rangle=\int_{0}^{t_{0}}\left\langle f_{i}\left(\boldsymbol{z}^{\varepsilon}\right), z_{i}^{\varepsilon}\right\rangle \leq C\left(\left\|\boldsymbol{z}^{\varepsilon}\right\|_{L^{2}\left(0, t_{0} ; L^{2}(\Omega)\right)^{M}}^{2}+1\right) .
$$

Here, we use the Lipschitz continuity of $f_{i}$ and the following elementary inequality

$$
2 b c \leq b^{2} \alpha+c^{2} / \alpha \text { for } b, c \in \mathbb{R} \text { and } \alpha>0 .
$$

This relation will often be used in the sequel. It follows from (3.2) that

$$
\begin{aligned}
\left\langle\nabla u_{i}^{\varepsilon}, \nabla z_{i}^{\varepsilon}\right\rangle= & \mu\left\|u_{i}^{\varepsilon}\right\|_{L^{2}(\Omega)}^{2}+\frac{\varepsilon}{2 \mu} \frac{\mathrm{d}}{\mathrm{d} t}\left\|\nabla v_{i}^{\varepsilon}\right\|_{L^{2}(\Omega)}^{2}+\left\langle\nabla \beta_{i}\left(\boldsymbol{z}^{\varepsilon}\right), \nabla z_{i}^{\varepsilon}\right\rangle-\mu\left\langle\nabla \beta_{i}\left(\boldsymbol{z}^{\varepsilon}\right), \nabla u_{i}^{\varepsilon}\right\rangle \\
= & \mu\left\|u_{i}^{\varepsilon}\right\|_{L^{2}(\Omega)}^{2}+\frac{\varepsilon}{2 \mu} \frac{\mathrm{d}}{\mathrm{d} t}\left\|\nabla v_{i}^{\varepsilon}\right\|_{L^{2}(\Omega)}^{2}+a\left\|\nabla z_{i}^{\varepsilon}\right\|_{L^{2}(\Omega)}^{2}+\left\langle\nabla \phi_{i}\left(\boldsymbol{z}^{\varepsilon}\right), \nabla z_{i}^{\varepsilon}\right\rangle \\
& -\mu a\left\langle\nabla z_{i}^{\varepsilon}, \nabla u_{i}^{\varepsilon}\right\rangle-\mu\left\langle\nabla \phi_{i}\left(\boldsymbol{z}^{\varepsilon}\right), \nabla u_{i}^{\varepsilon}\right\rangle .
\end{aligned}
$$

Here, $\phi_{i}(\boldsymbol{\eta})=\beta_{i}(\boldsymbol{\eta})-a \eta_{i}$ for all $\boldsymbol{\eta} \in \mathbb{R}^{M}$. The last term of the right-hand side of (3.7) can be estimated as follows:

$$
\left|\mu\left\langle\nabla \phi_{i}\left(\boldsymbol{z}^{\varepsilon}\right), \nabla u_{i}^{\varepsilon}\right\rangle\right| \leq \frac{\mu}{2}\left\|\nabla u_{i}^{\varepsilon}\right\|_{L^{2}(\Omega)}^{2}+\frac{\mu M L^{2}}{2} \sum_{j=1}^{M}\left\|\nabla z_{j}^{\varepsilon}\right\|_{L^{2}(\Omega)}^{2} .
$$

Collect the previous estimates and sum over $i$ from 1 to $M$ to get

$$
\begin{aligned}
\frac{1}{2}\left\|\boldsymbol{z}^{\varepsilon}\left(t_{0}\right)\right\|_{L^{2}(\Omega)^{M}}^{2} & +\frac{1}{1+\mu a}\left(a-\frac{\mu M^{2} L^{2}}{2}\right)\left\|\nabla \boldsymbol{z}^{\varepsilon}\right\|_{L^{2}\left(0, t_{0} ; L^{2}(\Omega)\right)}^{2}+\frac{\mu}{2(1+\mu a)}\left\|\nabla \boldsymbol{u}^{\varepsilon}\right\|_{L^{2}\left(0, t_{0} ; L^{2}(\Omega)\right)}^{2} \\
& +\frac{\varepsilon}{2 \mu(1+\mu a)}\left\|\nabla \boldsymbol{v}^{\varepsilon}\left(t_{0}\right)\right\|_{L^{2}(\Omega)}^{2}+\frac{1}{1+\mu a} \int_{0}^{t_{0}} \sum_{i=1}^{M}\left\langle\nabla \phi_{i}\left(\boldsymbol{z}^{\varepsilon}\right), \nabla z_{i}^{\varepsilon}\right\rangle \\
\leq & \frac{1}{2}\left\|\boldsymbol{z}_{0}^{\varepsilon}\right\|_{L^{2}(\Omega)^{M}}^{2}+\frac{\varepsilon}{2 \mu(1+\mu a)}\left\|\nabla \boldsymbol{v}_{0}^{\varepsilon}\right\|_{L^{2}(\Omega)}^{2}+C\left(\left\|\boldsymbol{z}^{\varepsilon}\right\|_{L^{2}\left(0, t_{0} ; L^{2}(\Omega)\right)^{M}}^{2}+1\right) .
\end{aligned}
$$

The last term of the left-hand side is positive due to the hypothesis (H2). Therefore, using (H4), (H6)' and the Gronwall inequality yields

$$
\left\|\boldsymbol{z}^{\varepsilon}\right\|_{\left(L^{\infty}\left(0, T ; L^{2}(\Omega)\right) \cap L^{2}\left(0, T ; H^{1}(\Omega)\right)\right)^{M}}+\left\|\nabla \boldsymbol{u}^{\varepsilon}\right\|_{L^{2}(Q)^{M}}+\left\|\nabla \boldsymbol{v}^{\varepsilon}\right\|_{L^{2}(Q)^{M}}+\sqrt{\varepsilon}\left\|\nabla \boldsymbol{v}^{\varepsilon}\right\|_{L^{\infty}\left(0, T ; L^{2}(\Omega)\right)^{M}} \leq C .
$$


Multiplying (3.2) by $\varepsilon v_{i}^{\varepsilon}$ and integrating both sides over space, we obtain

$$
\begin{aligned}
\frac{\varepsilon}{2} \frac{\mathrm{d}}{\mathrm{d} t}\left\|v_{i}^{\varepsilon}\right\|_{L^{2}(\Omega)}^{2} & =\mu\left\langle u_{i}^{\varepsilon}, v_{i}^{\varepsilon}\right\rangle-\mu\left\langle\beta_{i}\left(\boldsymbol{z}^{\varepsilon}\right), v_{i}^{\varepsilon}\right\rangle \\
& =\frac{1}{2}\left\|z_{i}^{\varepsilon}\right\|_{L^{2}(\Omega)}^{2}-\frac{\mu^{2}}{2}\left\|u_{i}^{\varepsilon}\right\|_{L^{2}(\Omega)}^{2}-\frac{1}{2}\left\|v_{i}^{\varepsilon}\right\|_{L^{2}(\Omega)}^{2}-\mu\left\langle\beta_{i}\left(\boldsymbol{z}^{\varepsilon}\right), v_{i}^{\varepsilon}\right\rangle \\
& \leq \frac{1}{2}\left\|z_{i}^{\varepsilon}\right\|_{L^{2}(\Omega)}^{2}-\frac{\mu^{2}}{2}\left\|u_{i}^{\varepsilon}\right\|_{L^{2}(\Omega)}^{2}-\frac{1}{4}\left\|v_{i}^{\varepsilon}\right\|_{L^{2}(\Omega)}^{2}+\mu^{2}\left\|\beta_{i}\left(\boldsymbol{z}^{\varepsilon}\right)\right\|_{L^{2}(\Omega)}^{2} .
\end{aligned}
$$

We deduce from $(\mathrm{H} 6)^{\prime}$ and (3.9) that

$$
\left\|\boldsymbol{u}^{\varepsilon}\right\|_{L^{2}(Q)^{M}}+\left\|\boldsymbol{v}^{\varepsilon}\right\|_{L^{2}(Q)^{M}}+\sqrt{\varepsilon}\left\|\boldsymbol{v}^{\varepsilon}\right\|_{L^{\infty}\left(0, T ; L^{2}(\Omega)\right)^{M}} \leq C .
$$

It follows from (3.4) and (3.9) that for $i=1, \ldots, M$ and $\varphi \in L^{2}\left(0, T ; H^{1}(\Omega)\right)$,

$$
\left|\int_{0}^{T}\left\langle\frac{\partial z_{i}^{\varepsilon}}{\partial t}, \varphi\right\rangle\right| \leq\left\|\nabla u_{i}^{\varepsilon}\right\|_{L^{2}(Q)}\|\nabla \varphi\|_{L^{2}(Q)}+\left\|f_{i}\left(\boldsymbol{z}^{\varepsilon}\right)\right\|_{L^{2}(Q)}\|\varphi\|_{L^{2}(Q)} \leq C\|\varphi\|_{L^{2}\left(0, T ; H^{1}(\Omega)\right)} .
$$

This means that

which completes the proof.

$$
\left\|\frac{\partial \boldsymbol{z}^{\varepsilon}}{\partial t}\right\|_{L^{2}\left(0, T ; H^{1}(\Omega)^{*}\right)^{M}} \leq C
$$

\subsection{Proof of Theorem 3.2}

In this section, we prove Theorem 3.2 .

Proof of Theorem 3.2. By virtue of Lemma 3.4 and the compactness of the embedding $L^{2}\left(0, T ; H^{1}(\Omega)\right) \cap$ $H^{1}\left(0, T ; H^{1}(\Omega)^{*}\right) \subset L^{2}(Q)\left([25]\right.$, Thm. 2.1), there exist subsequences $\left\{\boldsymbol{z}^{\varepsilon_{k}}\right\}$ and $\left\{\boldsymbol{u}^{\varepsilon_{k}}\right\}$ of $\left\{\boldsymbol{z}^{\varepsilon}\right\}$ and $\left\{\boldsymbol{u}^{\varepsilon}\right\}$ and functions $\boldsymbol{z}^{*}, \boldsymbol{u}^{*}$ such that

$$
\begin{aligned}
& \boldsymbol{z}^{*} \in\left(L^{\infty}\left(0, T ; L^{2}(\Omega)\right) \cap L^{2}\left(0, T ; H^{1}(\Omega)\right) \cap H^{1}\left(0, T ; H^{1}(\Omega)^{*}\right)\right)^{M}, \\
& \boldsymbol{u}^{*} \in L^{2}\left(0, T ; H^{1}(\Omega)\right)^{M}, \\
& \boldsymbol{z}^{\varepsilon_{k}} \rightarrow \boldsymbol{z}^{*} \quad \text { strongly in } L^{2}(Q)^{M} \text {, a.e. in } Q \text {, } \\
& \begin{array}{ll}
\boldsymbol{u}^{\varepsilon_{k}} \rightarrow \boldsymbol{u}^{*} \quad \text { and weakly in } L^{2}\left(0, T ; H^{1}(\Omega\right. \\
\text { weakly in } L^{2}\left(0, T ; H^{1}(\Omega)\right)^{M}
\end{array}
\end{aligned}
$$

as $\varepsilon_{k}$ tends to zero. The Lipschitz continuities of $\boldsymbol{\beta}$ and $\boldsymbol{f}$ imply that $\boldsymbol{\beta}\left(\boldsymbol{z}^{\varepsilon_{k}}\right)$ and $\boldsymbol{f}\left(\boldsymbol{z}^{\varepsilon_{k}}\right)$ also converge to $\boldsymbol{\beta}\left(\boldsymbol{z}^{*}\right)$ and $\boldsymbol{f}\left(\boldsymbol{z}^{*}\right)$ strongly in $L^{2}(Q)^{M}$ and a.e. in $Q$, respectively. Taking $\varphi_{i}=\varepsilon_{k} \zeta, \zeta \in C_{0}^{\infty}(Q)$ in (3.1) and letting to the limit in $\varepsilon_{k}$ yield

$$
\int_{0}^{T}\left\langle u_{i}^{*}-\beta_{i}\left(z^{*}\right), \zeta\right\rangle=0 \text { for all } \zeta \in C_{0}^{\infty}(Q)
$$

Therefore, $\boldsymbol{u}^{*}=\boldsymbol{\beta}\left(\boldsymbol{z}^{*}\right)$ holds a.e. in $Q$. From the initial condition (3.3), we have

$$
\int_{0}^{T}\left\langle\frac{\partial z_{i}^{\varepsilon}}{\partial t}, \varphi_{i}\right\rangle+\int_{0}^{T}\left\langle z_{i}^{\varepsilon}-\left(\mu u_{0 i}^{\varepsilon}+v_{0 i}^{\varepsilon}\right), \frac{\partial \varphi_{i}}{\partial t}\right\rangle=0
$$

for all functions $\varphi_{i} \in H^{1}(Q)$ with $\varphi_{i}(\cdot, T)=0$. Passing to the limit along the subsequences in (3.4) and (3.10), we observe that $\boldsymbol{z}^{*}$ is a weak solution of (1.1). Thus, the proof is complete. 


\section{Proof of Theorem 2.3}

We prove the following estimates for the solution $\left\{\boldsymbol{U}^{n}, \boldsymbol{V}^{n}\right\}$ of Problem 2.2 and a function $\boldsymbol{Z}^{n}=\mu \boldsymbol{U}^{n}+\boldsymbol{V}^{n}$.

Lemma 4.1. Suppose (H1)-(H4) and (H6) are satisfied. Then there exists a positive constant $C$ independent of $\varepsilon$ and $\tau$ such that

$$
\begin{aligned}
& \max _{0 \leq n \leq N_{T}}\left\|\boldsymbol{Z}^{n}\right\|_{L^{2}(\Omega)^{M}}^{2}+\tau \sum_{n=1}^{N_{T}}\left\|\boldsymbol{Z}^{n}\right\|_{H^{1}(\Omega)^{M}}^{2}+\tau \sum_{n=1}^{N_{T}}\left\|\bar{D}_{\tau} \boldsymbol{Z}^{n}\right\|_{\left(H^{1}(\Omega)^{*}\right)^{M}}^{2}+\tau^{2} \sum_{n=1}^{N_{T}}\left\|\bar{D}_{\tau} \boldsymbol{Z}^{n}\right\|_{L^{2}(\Omega)^{M}}^{2} \\
& \quad+\tau^{3} \sum_{n=1}^{N_{T}}\left\|\bar{D}_{\tau} \boldsymbol{Z}^{n}\right\|_{H^{1}(\Omega)^{M}}^{2}+\tau \sum_{n=1}^{N_{T}}\left\|\boldsymbol{U}^{n}\right\|_{H^{1}(\Omega)^{M}}^{2}+\tau^{2} \sum_{n=1}^{N_{T}}\left\|\Delta \boldsymbol{U}^{n}\right\|_{L^{2}(\Omega)^{M}}^{2}+\tau \sum_{n=1}^{N_{T}}\left\|\boldsymbol{V}^{n}\right\|_{H^{1}(\Omega)^{M}}^{2} \\
& \quad+\varepsilon \max _{0 \leq n \leq N_{T}}\left\|\boldsymbol{V}^{n}\right\|_{L^{2}(\Omega)^{M}}^{2}+(\varepsilon-\tau) \max _{0 \leq n \leq N_{T}}\left\|\boldsymbol{V}^{n}\right\|_{H^{1}(\Omega)^{M}}^{2}+\varepsilon^{2} \tau \sum_{n=1}^{N_{T}}\left\|\bar{D}_{\tau} \boldsymbol{V}^{n}\right\|_{L^{2}(\Omega)^{M}}^{2} \\
& \quad+(\varepsilon-\tau) \tau^{2} \sum_{n=1}^{N_{T}}\left\|\bar{D}_{\tau} \boldsymbol{V}^{n}\right\|_{H^{1}(\Omega)^{M}}^{2} \leq C .
\end{aligned}
$$

In this section, we denote by $C$ a generic positive constant independent of $\varepsilon$ and $\tau$.

This lemma implies that the linear scheme (1.10) is stable while $\tau \leq \varepsilon$. A better numerical solution may be obtained when $\varepsilon$ is taken to be small because the solution of (1.9) is expected to be close to that of (1.1) while $\varepsilon$ is small. Therefore, $\varepsilon=\tau$ is the best choice.

Proof. Note that (2.1) and (2.2) can be combined to give

$$
\left\langle Z_{i}^{n}-Z_{i}^{n-1}, \varphi\right\rangle+\tau\left\langle\nabla U_{i}^{n}, \nabla \varphi\right\rangle=\tau\left\langle f_{i}\left(\boldsymbol{Z}^{n-1}\right), \varphi\right\rangle
$$

for all $\varphi \in H^{1}(\Omega)$. Take $\varphi=Z_{i}^{n}$ to get

$$
\left\langle Z_{i}^{n}-Z_{i}^{n-1}, Z_{i}^{n}\right\rangle+\tau\left\langle\nabla U_{i}^{n}, \nabla Z_{i}^{n}\right\rangle=\tau\left\langle f_{i}\left(\boldsymbol{Z}^{n-1}\right), Z_{i}^{n}\right\rangle
$$

Using the elementary relations $2 b(b-c)=b^{2}-c^{2}+(b-c)^{2}$ for $b, c \in \mathbb{R}$ and (3.6), the first term of the left-hand side and the right-hand side can be estimated as follows:

$$
\begin{aligned}
2\left\langle Z_{i}^{n}-Z_{i}^{n-1}, Z_{i}^{n}\right\rangle & =\left\|Z_{i}^{n}\right\|_{L^{2}(\Omega)}^{2}-\left\|Z_{i}^{n-1}\right\|_{L^{2}(\Omega)}^{2}+\left\|Z_{i}^{n}-Z_{i}^{n-1}\right\|_{L^{2}(\Omega)}^{2}, \\
\tau\left\langle f_{i}\left(\boldsymbol{Z}^{n-1}\right), Z_{i}^{n}\right\rangle & \leq \frac{\tau}{2}\left\|f_{i}\left(\boldsymbol{Z}^{n-1}\right)\right\|_{L^{2}(\Omega)}^{2}+\frac{\tau}{2}\left\|Z_{i}^{n}\right\|_{L^{2}(\Omega)}^{2} .
\end{aligned}
$$

One of the key points of the proof of Lemma 3.4 was that 1 is divided into $1 /(1+\mu a)$ and $\mu a /(1+\mu a)$ in order to estimate $\left\langle\nabla u_{i}^{\varepsilon}, \nabla z_{i}^{\varepsilon}\right\rangle$. Hence, we estimate the second term of the left-hand side of (4.2) twice in different ways. It follows from (2.2) that

$$
\begin{aligned}
U_{i}^{n} & =U_{i}^{n}-U_{i}^{n-1}+\beta_{i}\left(\boldsymbol{Z}^{n-1}\right)+\frac{\varepsilon}{\mu} \bar{D}_{\tau} V_{i}^{n} \\
& =\frac{Z_{i}^{n}-Z_{i}^{n-1}}{\mu}+\beta_{i}\left(\boldsymbol{Z}^{n-1}\right)+\frac{1}{\mu}(\varepsilon-\tau) \bar{D}_{\tau} V_{i}^{n} .
\end{aligned}
$$


Therefore, we obtain

$$
\begin{gathered}
\tau\left\langle\nabla U_{i}^{n}, \nabla Z_{i}^{n}\right\rangle=\mu \tau\left\|\nabla U_{i}^{n}\right\|_{L^{2}(\Omega)}^{2}+\frac{1}{\mu} \tau\left\langle\nabla\left(Z_{i}^{n}-Z_{i}^{n-1}\right), \nabla Z_{i}^{n}\right\rangle-\tau\left\langle\nabla\left(Z_{i}^{n}-Z_{i}^{n-1}\right), \nabla U_{i}^{n}\right\rangle \\
+\tau\left\langle\nabla \beta_{i}\left(\boldsymbol{Z}^{n-1}\right), \nabla V_{i}^{n}\right\rangle+\frac{1}{\mu}(\varepsilon-\tau)\left\langle\nabla\left(V_{i}^{n}-V_{i}^{n-1}\right), \nabla V_{i}^{n}\right\rangle \\
=\mu \tau\left\|\nabla U_{i}^{n}\right\|_{L^{2}(\Omega)}^{2}+\frac{1}{2 \mu} \tau\left(\left\|\nabla Z_{i}^{n}\right\|_{L^{2}(\Omega)}^{2}-\left\|\nabla Z_{i}^{n-1}\right\|_{L^{2}(\Omega)}^{2}+\left\|\nabla\left(Z_{i}^{n}-Z_{i}^{n-1}\right)\right\|_{L^{2}(\Omega)}^{2}\right) \\
\quad-\tau\left\langle\nabla\left(Z_{i}^{n}-Z_{i}^{n-1}\right), \nabla U_{i}^{n}\right\rangle+a \tau\left\|\nabla Z_{i}^{n-1}\right\|_{L^{2}(\Omega)}^{2}+\tau\left\langle\nabla \phi_{i}\left(\boldsymbol{Z}^{n-1}\right), \nabla Z_{i}^{n-1}\right\rangle \\
\quad+a \tau\left\langle\nabla Z_{i}^{n-1}, \nabla\left(Z_{i}^{n}-Z_{i}^{n-1}\right)\right\rangle+\tau\left\langle\nabla \phi_{i}\left(\boldsymbol{Z}^{n-1}\right), \nabla\left(Z_{i}^{n}-Z_{i}^{n-1}\right)\right\rangle-\mu a \tau\left\langle\nabla Z_{i}^{n-1}, \nabla U_{i}^{n}\right\rangle \\
-\mu \tau\left\langle\nabla \phi_{i}\left(\boldsymbol{Z}^{n-1}\right), \nabla U_{i}^{n}\right\rangle+\frac{1}{2 \mu}(\varepsilon-\tau)\left(\left\|\nabla V_{i}^{n}\right\|_{L^{2}(\Omega)}^{2}-\left\|\nabla V_{i}^{n-1}\right\|_{L^{2}(\Omega)}^{2}+\left\|\nabla\left(V_{i}^{n}-V_{i}^{n-1}\right)\right\|_{L^{2}(\Omega)}^{2}\right) .
\end{gathered}
$$

On the other hand, we have

$$
\tau\left\langle\nabla U_{i}^{n}, \nabla Z_{i}^{n}\right\rangle=\tau\left\langle\nabla U_{i}^{n}, \nabla\left(Z_{i}^{n}-Z_{i}^{n-1}\right)\right\rangle+\tau\left\langle\nabla U_{i}^{n}, \nabla Z_{i}^{n-1}\right\rangle .
$$

Add up (4.3) and (4.4) weighted by $\mu a$ and sum over $n$ from 1 to $m \leq N_{T}$. Then, summation by parts yields

$$
\begin{gathered}
(1+\mu a) \tau \sum_{n=1}^{m}\left\langle\nabla U_{i}^{n}, \nabla Z_{i}^{n}\right\rangle=\mu \tau \sum_{n=1}^{m}\left\|\nabla U_{i}^{n}\right\|_{L^{2}(\Omega)}^{2}+\frac{1}{2}\left(\frac{1}{\mu}+a\right) \tau\left(\left\|\nabla Z_{i}^{m}\right\|_{L^{2}(\Omega)}^{2}-\left\|\nabla Z_{i}^{0}\right\|_{L^{2}(\Omega)}^{2}\right) \\
+\frac{1}{2}\left(\frac{1}{\mu}-a\right) \sum_{n=1}^{m}\left\|\nabla\left(Z_{i}^{n}-Z_{i}^{n-1}\right)\right\|_{L^{2}(\Omega)}^{2}-(1-\mu a) \tau \sum_{n=1}^{m}\left\langle\nabla\left(Z_{i}^{n}-Z_{i}^{n-1}\right), \nabla U_{i}^{n}\right\rangle+a \tau \sum_{n=0}^{m-1}\left\|\nabla Z_{i}^{n}\right\|_{L^{2}(\Omega)}^{2} \\
+\tau \sum_{n=0}^{m-1}\left\langle\nabla \phi_{i}\left(\boldsymbol{Z}^{n}\right), \nabla Z_{i}^{n}\right\rangle+\tau \sum_{n=1}^{m}\left\langle\nabla \phi_{i}\left(\boldsymbol{Z}^{n-1}\right), \nabla\left(Z_{i}^{n}-Z_{i}^{n-1}\right)\right\rangle-\mu \tau \sum_{n=1}^{m}\left\langle\nabla \phi_{i}\left(\boldsymbol{Z}^{n-1}\right), \nabla U_{i}^{n}\right\rangle \\
+\frac{1}{2 \mu}(\varepsilon-\tau)\left(\left\|\nabla V_{i}^{m}\right\|_{L^{2}(\Omega)}^{2}-\left\|\nabla V_{i}^{0}\right\|_{L^{2}(\Omega)}^{2}+\sum_{n=1}^{m}\left\|\nabla\left(V_{i}^{n}-V_{i}^{n-1}\right)\right\|_{L^{2}(\Omega)}^{2}\right)
\end{gathered}
$$

Note that $\mu a<1$ and $U_{i}^{n} \in H^{3}(\Omega)$. In view of (4.1) and (3.6), we can estimate as follows:

$$
\begin{gathered}
-(1-\mu a) \tau \sum_{n=1}^{m}\left\langle\nabla\left(Z_{i}^{n}-Z_{i}^{n-1}\right), \nabla U_{i}^{n}\right\rangle=(1-\mu a) \tau^{2} \sum_{n=1}^{m}\left(\left\|\Delta U_{i}^{n}\right\|_{L^{2}(\Omega)}^{2}+\left\langle f_{i}\left(\boldsymbol{Z}^{n-1}\right), \Delta U_{i}^{n}\right\rangle\right) \\
\geq \frac{1-\mu a}{2} \tau^{2} \sum_{n=1}^{m}\left\|\Delta U_{i}^{n}\right\|_{L^{2}(\Omega)}^{2}-\frac{1-\mu a}{2} \tau^{2} \sum_{n=0}^{m-1}\left\|f_{i}\left(\boldsymbol{Z}^{n}\right)\right\|_{L^{2}(\Omega)}^{2}, \\
\leq \frac{2 \mu M L^{2}}{3(1-\mu a)} \tau \sum_{n=0}^{m-1} \sum_{j=1}^{M}\left\|\nabla Z_{j}^{n}\right\|_{L^{2}(\Omega)}^{2}+\frac{3(1-\mu a)}{8 \mu} \tau \sum_{n=1}^{m}\left\|\nabla\left(Z_{i}^{n}-Z_{i}^{n-1}\right)\right\|_{L^{2}(\Omega)}^{2}, \\
\left|\tau \sum_{n=1}^{m}\left\langle\nabla \phi_{i}\left(\boldsymbol{Z}^{n-1}\right), \nabla\left(Z_{i}^{n}-Z_{i}^{n-1}\right)\right\rangle\right| \leq \frac{2 \mu}{3(1-\mu a)} \tau \sum_{n=0}^{m-1}\left\|\nabla \phi_{i}\left(\boldsymbol{Z}^{n}\right)\right\|_{L^{2}(\Omega)}^{2}+\frac{3(1-\mu a)}{8 \mu} \tau \sum_{n=1}^{m}\left\|\nabla\left(Z_{i}^{n}-Z_{i}^{n-1}\right)\right\|_{L^{2}(\Omega)}^{2} \\
\left|\mu \tau \sum_{n=1}^{m}\left\langle\nabla \phi_{i}\left(\boldsymbol{Z}^{n-1}\right), \nabla U_{i}^{n}\right\rangle\right| \leq \frac{\mu M L^{2}}{3} \tau \sum_{n=0}^{m-1} \sum_{j=1}^{M}\left\|\nabla Z_{j}^{n}\right\|_{L^{2}(\Omega)}^{2}+\frac{3 \mu}{4} \tau \sum_{n=1}^{m}\left\|\nabla U_{i}^{n}\right\|_{L^{2}(\Omega)}^{2} .
\end{gathered}
$$


Collecting all the previous bounds and summing over $i$ from 1 to $M$, we obtain

$$
\begin{aligned}
& \frac{1}{2}\left\|\boldsymbol{Z}^{m}\right\|_{L^{2}(\Omega)^{M}}^{2}+\frac{1}{2} \sum_{n=1}^{m}\left\|\boldsymbol{Z}^{n}-\boldsymbol{Z}^{n-1}\right\|_{L^{2}(\Omega)^{M}}^{2}+\frac{\mu}{4(1+\mu a)} \tau \sum_{n=1}^{m}\left\|\nabla \boldsymbol{U}^{n}\right\|_{L^{2}(\Omega)^{M}}^{2}+\frac{1}{2 \mu} \tau\left\|\nabla \boldsymbol{Z}^{m}\right\|_{L^{2}(\Omega)^{M}}^{2} \\
& \quad+\frac{1-\mu a}{8 \mu(1+\mu a)} \tau \sum_{n=1}^{m}\left\|\nabla\left(\boldsymbol{Z}^{n}-\boldsymbol{Z}^{n-1}\right)\right\|_{L^{2}(\Omega)^{M}}^{2}+\frac{1-\mu a}{2(1+\mu a)} \tau^{2} \sum_{n=1}^{m}\left\|\Delta \boldsymbol{U}^{n}\right\|_{L^{2}(\Omega)^{M}}^{2} \\
& \quad+\frac{1}{1+\mu a}\left(a-\frac{\mu(3-\mu a) M^{2} L^{2}}{3(1-\mu a)}\right) \tau \sum_{n=0}^{m-1}\left\|\nabla \boldsymbol{Z}^{n}\right\|_{L^{2}(\Omega)^{M}}^{2}+\frac{1}{1+\mu a} \tau \sum_{n=0}^{m-1} \sum_{i=1}^{M}\left\langle\nabla \phi_{i}\left(\boldsymbol{Z}^{n}\right), \nabla Z_{i}^{n}\right\rangle \\
& \quad+\frac{1}{2 \mu(1+\mu a)}(\varepsilon-\tau)\left(\left\|\nabla \boldsymbol{V}^{m}\right\|_{L^{2}(\Omega)^{M}}^{2}+\sum_{n=1}^{m}\left\|\nabla\left(\boldsymbol{V}^{n}-\boldsymbol{V}^{n-1}\right)\right\|_{L^{2}(\Omega)^{M}}^{2}\right) \\
& \leq C\left(1+\tau \sum_{n=0}^{m}\left\|\boldsymbol{Z}^{n}\right\|_{L^{2}(\Omega)^{M}}^{2}+\left\|\boldsymbol{Z}^{0}\right\|_{L^{2}(\Omega)}^{2}+\frac{1}{2 \mu} \tau\left\|\nabla \boldsymbol{Z}^{0}\right\|_{L^{2}(\Omega)^{M}}^{2}+(\varepsilon-\tau)\left\|\nabla \boldsymbol{V}^{0}\right\|_{L^{2}(\Omega)^{M}}^{2}\right) .
\end{aligned}
$$

By virtue of (H2) and (H4), the seventh and eighth terms are positive. We deduce from (H6) and the discrete Gronwall inequality that

$$
\begin{aligned}
& \max _{0 \leq n \leq N_{T}}\left\|\boldsymbol{Z}^{n}\right\|_{L^{2}(\Omega)^{M}}^{2}+\tau \sum_{n=1}^{N_{T}}\left\|\boldsymbol{Z}^{n}\right\|_{H^{1}(\Omega)^{M}}^{2}+\tau^{2} \sum_{n=1}^{N_{T}}\left\|\bar{D}_{\tau} \boldsymbol{Z}^{n}\right\|_{L^{2}(\Omega)^{M}}^{2}+\tau^{3} \sum_{n=1}^{N_{T}}\left\|\bar{D}_{\tau} \boldsymbol{Z}^{n}\right\|_{H^{1}(\Omega)^{M}}^{2} \\
& \quad+\tau \sum_{n=1}^{N_{T}}\left\|\nabla \boldsymbol{U}^{n}\right\|_{L^{2}(\Omega)^{M}}^{2}+\tau^{2} \sum_{n=1}^{N_{T}}\left\|\Delta \boldsymbol{U}^{n}\right\|_{L^{2}(\Omega)^{M}}^{2}+\tau \sum_{n=1}^{N_{T}}\left\|\nabla \boldsymbol{V}^{n}\right\|_{L^{2}(\Omega)^{M}}^{2} \\
& \quad+(\varepsilon-\tau) \max _{0 \leq n \leq N_{T}}\left\|\nabla \boldsymbol{V}^{n}\right\|_{L^{2}(\Omega)^{M}}^{2}+(\varepsilon-\tau) \tau^{2} \sum_{n=1}^{N_{T}}\left\|\nabla \bar{D}_{\tau} \boldsymbol{V}^{n}\right\|_{L^{2}(\Omega)^{M}}^{2} \leq C .
\end{aligned}
$$

Multiplying (2.2) by $2 \varepsilon \tau V_{i}^{n}$, we have

$$
\begin{aligned}
& \varepsilon\left(\left\|V_{i}^{n}\right\|_{L^{2}(\Omega)}^{2}-\left\|V_{i}^{n-1}\right\|_{L^{2}(\Omega)}^{2}+\left\|V_{i}^{n}-V_{i}^{n-1}\right\|_{L^{2}(\Omega)}^{2}\right) \\
& \quad=2 \mu \tau\left\langle U_{i}^{n-1}, V_{i}^{n-1}\right\rangle+2 \mu \tau\left\langle U_{i}^{n-1}, V_{i}^{n}-V_{i}^{n-1}\right\rangle-2 \mu \tau\left\langle\beta_{i}\left(\boldsymbol{Z}^{n-1}\right), V_{i}^{n}\right\rangle \\
& \quad=\tau\left\|Z_{i}^{n-1}\right\|_{L^{2}(\Omega)}^{2}-\mu^{2} \tau\left\|U_{i}^{n-1}\right\|_{L^{2}(\Omega)}^{2}-\tau\left\|V_{i}^{n-1}\right\|_{L^{2}(\Omega)}^{2}+2 \mu \tau\left\langle U_{i}^{n-1}, V_{i}^{n}-V_{i}^{n-1}\right\rangle-2 \mu \tau\left\langle\beta_{i}\left(\boldsymbol{Z}^{n-1}\right), V_{i}^{n}\right\rangle .
\end{aligned}
$$

Sum over $n$ from 1 to $m \leq N_{T}$ and apply (3.6) to get

$$
\begin{aligned}
\frac{\varepsilon}{2}\left\|V_{i}^{m}\right\|_{L^{2}(\Omega)}^{2}+(\varepsilon-\tau) \sum_{n=1}^{m}\left\|V_{i}^{n}-V_{i}^{n-1}\right\|_{L^{2}(\Omega)}^{2}+ & \tau \sum_{n=0}^{m-1}\left\|V_{i}^{n}\right\|_{L^{2}(\Omega)}^{2} \\
& \leq \varepsilon\left\|V_{i}^{0}\right\|_{L^{2}(\Omega)}^{2}+\tau \sum_{n=0}^{m-1}\left\|Z_{i}^{n}\right\|_{L^{2}(\Omega)}^{2}+2 \mu^{2} \tau \sum_{n=0}^{m-1}\left\|\beta_{i}\left(\boldsymbol{Z}^{n}\right)\right\|_{L^{2}(\Omega)}^{2} .
\end{aligned}
$$

The assumption (H6) and the estimate (4.6) imply

$$
\varepsilon \max _{0 \leq n \leq N_{T}}\left\|\boldsymbol{V}^{n}\right\|_{L^{2}(\Omega)^{M}}^{2}+\tau \sum_{n=1}^{N_{T}}\left\|\boldsymbol{V}^{n}\right\|_{L^{2}(\Omega)^{M}}^{2}+\tau \sum_{n=1}^{N_{T}}\left\|\boldsymbol{U}^{n}\right\|_{L^{2}(\Omega)^{M}}^{2} \leq C .
$$


Multiplying (2.2) by $\tau \bar{D}_{\tau} V_{i}^{n}$ and summing the result over all $n \in\left\{1,2, \ldots, N_{T}\right\}$ yield

$$
\tau \sum_{n=1}^{N_{T}}\left\|\bar{D}_{\tau} V_{i}^{n}\right\|_{L^{2}(\Omega)}^{2} \leq \frac{2 \mu^{2}}{\varepsilon^{2}} \tau \sum_{n=0}^{N_{T}-1}\left(\left\|U_{i}^{n}\right\|_{L^{2}(\Omega)}^{2}+\left\|\beta_{i}\left(\boldsymbol{Z}^{n}\right)\right\|_{L^{2}(\Omega)}^{2}\right)
$$

Therefore, we obtain

$$
\varepsilon^{2} \tau \sum_{n=1}^{N_{T}}\left\|\bar{D}_{\tau} \boldsymbol{V}^{n}\right\|_{L^{2}(\Omega)^{M}}^{2} \leq C
$$

We deduce from (4.1) that

$$
\tau \sum_{n=1}^{N_{T}}\left|\left\langle\bar{D}_{\tau} Z_{i}^{n}, \varphi\right\rangle\right|^{2} \leq 2 \tau \sum_{n=1}^{N_{T}}\left\|\nabla U_{i}^{n}\right\|_{L^{2}(\Omega)}^{2}\|\nabla \varphi\|_{L^{2}(\Omega)}^{2}+2 \tau \sum_{n=1}^{N_{T}}\left\|f_{i}\left(\boldsymbol{Z}^{n-1}\right)\right\|_{L^{2}(\Omega)}^{2}\|\varphi\|_{L^{2}(\Omega)}^{2} \leq C\|\varphi\|_{H^{1}(\Omega)}^{2}
$$

for all $\varphi \in H^{1}(\Omega)$, which implies

$$
\tau \sum_{n=1}^{N_{T}}\left\|\bar{D}_{\tau} \boldsymbol{Z}^{n}\right\|_{\left(H^{1}(\Omega)^{*}\right)^{M}}^{2} \leq C
$$

Thus, the proof is complete.

We denote by $\widehat{\boldsymbol{Z}}^{(\varepsilon, \tau)}$ the function obtained by linear interpolation in time of $\left\{\boldsymbol{Z}^{n}\right\}$, and by $\boldsymbol{Z}^{(\varepsilon, \tau)}$ the piecewise constant function in time; namely,

$$
\widehat{Z}_{i}^{(\varepsilon, \tau)}(\cdot, t)=Z_{i}^{n-1}+\frac{t-(n-1) \tau}{\tau}\left(Z_{i}^{n}-Z_{i}^{n-1}\right)
$$

for $t \in((n-1) \tau, n \tau], n=1,2, \ldots, N_{T}$ and $i=1,2, \ldots, M$,

$$
Z_{i}^{(\varepsilon, \tau)}(\cdot, t)=Z_{i}^{n}
$$

for $t \in((n-1) \tau, n \tau], n=0,1,2, \ldots, N_{T}$ and $i=1,2, \ldots, M$. Functions $\widehat{\boldsymbol{U}}^{(\varepsilon, \tau)}, \widehat{\boldsymbol{V}}^{(\varepsilon, \tau)}, \boldsymbol{U}^{(\varepsilon, \tau)}$ and $\boldsymbol{V}^{(\varepsilon, \tau)}$ are similarly defined. Then, (2.1), (2.2) and (4.1) can be rewritten in the form

$$
\begin{aligned}
& \int_{0}^{T}\left\langle\frac{\partial \widehat{U}_{i}^{(\varepsilon, \tau)}}{\partial t}(t), \varphi(t)\right\rangle \mathrm{d} t+\frac{1}{\mu} \int_{0}^{T}\left\langle\nabla U_{i}^{(\varepsilon, \tau)}(t), \nabla \varphi(t)\right\rangle \mathrm{d} t \\
& \quad=\frac{1}{\mu} \int_{0}^{T}\left\langle f_{i}\left(\boldsymbol{Z}^{(\varepsilon, \tau)}(t-\tau)\right), \varphi(t)\right\rangle \mathrm{d} t-\frac{1}{\varepsilon} \int_{0}^{T}\left\langle U_{i}^{(\varepsilon, \tau)}(t-\tau)-\beta_{i}\left(\boldsymbol{Z}_{i}^{(\varepsilon, \tau)}(t-\tau)\right), \varphi(t)\right\rangle \mathrm{d} t \\
& \int_{0}^{T}\left\langle\frac{\partial \widehat{V}_{i}^{(\varepsilon, \tau)}}{\partial t}(t), \psi(t)\right\rangle \mathrm{d} t=\frac{\mu}{\varepsilon} \int_{0}^{T}\left\langle U_{i}^{(\varepsilon, \tau)}(t-\tau)-\beta_{i}\left(\boldsymbol{Z}_{i}^{(\varepsilon, \tau)}(t-\tau)\right), \psi(t)\right\rangle \mathrm{d} t \\
& \int_{0}^{T}\left\langle\frac{\partial \widehat{Z}_{i}^{(\varepsilon, \tau)}}{\partial t}(t), \varphi(t)\right\rangle \mathrm{d} t+\int_{0}^{T}\left\langle\nabla U_{i}^{(\varepsilon, \tau)}(t), \nabla \varphi(t)\right\rangle \mathrm{d} t=\int_{0}^{T}\left\langle f_{i}\left(\boldsymbol{Z}^{(\varepsilon, \tau)}(t-\tau)\right), \varphi(t)\right\rangle \mathrm{d} t
\end{aligned}
$$

for all $\varphi \in L^{2}\left(0, T ; H^{1}(\Omega)\right)$ and $\psi \in L^{2}(Q), i=1,2, \ldots, M$. 
Proof of Theorem 2.3. The proof is analogous to the proof of Theorem 3.2. Lemma 4.1 implies

$$
\begin{array}{r}
\left\|\widehat{\boldsymbol{Z}}^{(\varepsilon, \tau)}\right\|_{\left(L^{\infty}\left(0, T ; L^{2}(\Omega)\right) \cap L^{2}\left(0, T ; H^{1}(\Omega)\right) \cap H^{1}\left(0, T ; H^{1}(\Omega)^{*}\right)\right)^{M}}+\left\|\boldsymbol{Z}^{(\varepsilon, \tau)}\right\|_{\left(L^{\infty}\left(0, T ; L^{2}(\Omega)\right) \cap L^{2}\left(0, T ; H^{1}(\Omega)\right)\right)^{M}} \\
+\left\|\boldsymbol{U}^{(\varepsilon, \tau)}\right\|_{L^{2}\left(0, T ; H^{1}(\Omega)\right)^{M}}+\left\|\boldsymbol{V}^{(\varepsilon, \tau)}\right\|_{L^{2}\left(0, T ; H^{1}(\Omega)\right)^{M}} \leq C .
\end{array}
$$

Here, $C$ is independent of $\varepsilon$ and $\tau$. Therefore, there exist subsequences $\left\{\widehat{\boldsymbol{Z}}^{\left(\varepsilon_{k}, \tau_{k}\right)}\right\},\left\{\boldsymbol{Z}^{\left(\varepsilon_{k}, \tau_{k}\right)}\right\}$ and $\left\{\boldsymbol{U}^{\left(\varepsilon_{k}, \tau_{k}\right)}\right\}$ of $\left\{\widehat{\boldsymbol{Z}}^{(\varepsilon, \tau)}\right\},\left\{\boldsymbol{Z}^{(\varepsilon, \tau)}\right\}$ and $\left\{\boldsymbol{U}^{(\varepsilon, \tau)}\right\}$, respectively, and functions $\boldsymbol{z}^{*}, \boldsymbol{u}^{*}$ such that

$$
\begin{aligned}
& \boldsymbol{z}^{*} \in\left(L^{\infty}\left(0, T ; L^{2}(\Omega)\right) \cap L^{2}\left(0, T ; H^{1}(\Omega)\right) \cap H^{1}\left(0, T ; H^{1}(\Omega)^{*}\right)\right)^{M}, \\
& \boldsymbol{u}^{*} \in L^{2}\left(0, T ; H^{1}(\Omega)\right)^{M},
\end{aligned}
$$

$$
\begin{array}{ll}
\widehat{\boldsymbol{Z}}^{(\varepsilon, \tau)} \rightarrow \boldsymbol{z}^{*} & \text { strongly in } L^{2}(Q)^{M}, \text { a.e. in } Q, \\
& \text { and weakly in } L^{2}\left(0, T ; H^{1}(\Omega)\right)^{M} \text { and } H^{1}\left(0, T ; H^{1}(\Omega)^{*}\right)^{M}, \\
\boldsymbol{Z}^{(\varepsilon, \tau)}, \boldsymbol{Z}^{(\varepsilon, \tau)}(\cdot, \cdot-\tau) \rightarrow \boldsymbol{z}^{*} & \text { strongly in } L^{2}(Q)^{M}, \text { a.e. in } Q, \text { and weakly in } L^{2}\left(0, T ; H^{1}(\Omega)\right)^{M}, \\
\boldsymbol{U}^{(\varepsilon, \tau)}, \boldsymbol{U}^{(\varepsilon, \tau)}(\cdot, \cdot-\tau) \rightarrow \boldsymbol{u}^{*} & \text { weakly in } L^{2}\left(0, T ; H^{1}(\Omega)\right)^{M}
\end{array}
$$

as $\varepsilon_{k}, \tau_{k} \rightarrow 0$. Noticing (4.9) and using the same strategy as in the proof of Theorem 3.2, we find that $\boldsymbol{u}^{*}=\boldsymbol{\beta}\left(\boldsymbol{z}^{*}\right)$ and $\boldsymbol{z}^{*}$ is a weak solution of (1.1).

Remark 4.2. It follows from Lemma 4.1 that for each $\varepsilon$, there exists a positive constant $C$ which does not depend on sufficiently small $\tau$ such that

$$
\begin{array}{r}
\left\|\widehat{\boldsymbol{U}}^{(\varepsilon, \tau)}\right\|_{\left(L^{\infty}\left(0, T ; L^{2}(\Omega)\right) \cap L^{2}\left(0, T ; H^{1}(\Omega)\right) \cap H^{1}\left(0, T ; H^{1}(\Omega) *\right)\right)^{M}}+\left\|\boldsymbol{U}^{(\varepsilon, \tau)}\right\|_{\left(L^{\infty}\left(0, T ; L^{2}(\Omega)\right) \cap L^{2}\left(0, T ; H^{1}(\Omega)\right)\right)^{M}} \\
+\left\|\widehat{\boldsymbol{V}}^{(\varepsilon, \tau)}\right\|_{\left(L^{\infty}\left(0, T ; H^{1}(\Omega)\right) \cap H^{1}(Q)\right)^{M}}+\left\|\boldsymbol{V}^{(\varepsilon, \tau)}\right\|_{\left(L^{\infty}\left(0, T ; H^{1}(\Omega)\right)\right)^{M}} \leq C .
\end{array}
$$

Hence, passing to the limit in $\tau$ along subsequences in (4.7) and (4.8), we can prove the existence of the weak solution of (1.9).

\section{NUMERICAL EXPERIMENTS}

In this section, we give some numerical results in one and two space dimensions to demonstrate the effectiveness of our scheme.

\subsection{D experiments}

We deal with a problem treated numerically by Galiano et al. [7] and by Barrett and Blowey [1]. To this end, we introduce the following cross-diffusion-convection system.

$$
\begin{cases}\frac{\partial z_{i}}{\partial t}=\Delta \beta_{i}(\boldsymbol{z})+d_{i} \operatorname{div}\left(z_{i} \nabla p\right) & \text { in } \quad Q \\ \frac{\partial \beta_{i}(\boldsymbol{z})}{\partial \nu}+d_{i} z_{i} \frac{\partial p}{\partial \nu}=0 & \text { on } \quad \partial \Omega \times(0, T) \\ z_{i}(\cdot, 0)=z_{0 i} & \text { in } \quad \Omega\end{cases}
$$


for $i=1,2, \ldots, M$, where $d_{i}$ are non-negative constants and $p$ is a given function. The following linear scheme is proposed to approximate the solution of (5.1). Set $Z_{i}^{0}=\mu u_{0 i}^{\varepsilon, \tau}+v_{0 i}^{\varepsilon, \tau}$ and calculate

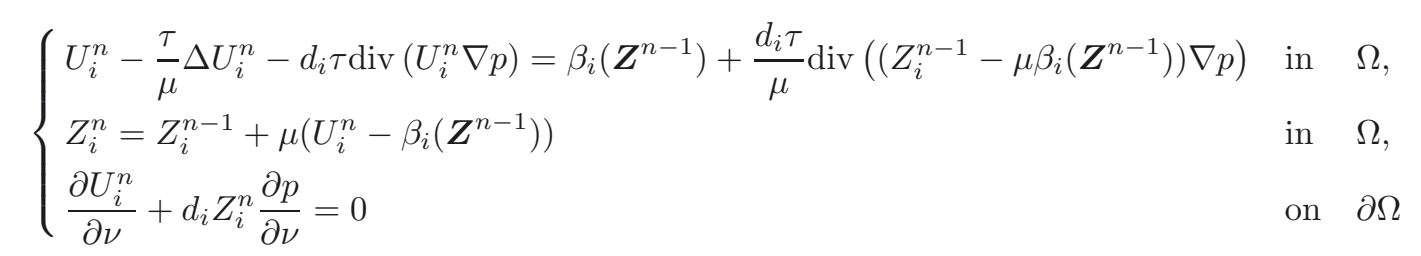

for $i=1,2, \ldots, M, n=1,2, \ldots, N_{T}$. The convection terms $\operatorname{div}\left(z_{i} \nabla p\right)$ are calculated $\operatorname{by} \operatorname{div}\left(Z_{i}^{n} \nabla p\right)=$ $\operatorname{div}\left(\left(Z_{i}^{n-1}+\mu\left(U_{i}^{n}-\beta_{i}\left(\boldsymbol{Z}^{n-1}\right)\right)\right) \nabla p\right)$. We can also choose $\operatorname{div}\left(Z_{i}^{n-1} \nabla p\right)$ as the time discretization of the convection terms. As a consequence of our analyses, we obtain convergence results for (5.2) as well as the corresponding reaction-diffusion-convection system

$$
\begin{cases}\frac{\partial u_{i}}{\partial t}=\frac{1}{\mu} \Delta u_{i}-\frac{1}{\varepsilon}\left(u_{i}-\beta_{i}(\mu \boldsymbol{u}+\boldsymbol{v})\right)+\frac{d_{i}}{\mu} \operatorname{div}\left(\left(\mu u_{i}+v_{i}\right) \nabla p\right) & \text { in } \quad Q \\ \frac{\partial v_{i}}{\partial t}=\frac{\mu}{\varepsilon}\left(u_{i}-\beta_{i}(\mu \boldsymbol{u}+\boldsymbol{v})\right) & \text { in } \quad Q \\ \frac{\partial u_{i}}{\partial \nu}+d_{i}\left(\mu u_{i}+v_{i}\right) \frac{\partial p}{\partial \nu}=0 & \text { on } \quad \partial \Omega \times(0, T) \\ u_{i}(\cdot, 0)=u_{0 i}^{\varepsilon}, \quad v_{i}(\cdot, 0)=v_{0 i}^{\varepsilon} & \text { in } \Omega\end{cases}
$$

for $i=1,2, \ldots, M$.

Corollary 5.1. Assume that $(\mathrm{H} 1),(\mathrm{H} 2)^{\prime},(\mathrm{H} 4)-(\mathrm{H} 6)$ and $(\mathrm{H} 6)^{\prime}$ are satisfied. Suppose $p \in W^{2, \infty}(\Omega)$. Let $\left(\boldsymbol{u}^{\varepsilon}, \boldsymbol{v}^{\varepsilon}\right)$ be the weak solution of (5.3) and $\left\{\boldsymbol{Z}^{n}, \boldsymbol{U}^{n}\right\}_{n=0}^{N_{T}}$ be that of (5.2). We denote by $\boldsymbol{Z}^{(\tau)}$ and $\boldsymbol{U}^{(\tau)}$ the functions obtained by piecewise constant interpolation in time of $\left\{\boldsymbol{Z}^{n}\right\}$ and $\left\{\boldsymbol{U}^{n}\right\}$, respectively. Then, there exist subsequences $\left\{\boldsymbol{u}^{\varepsilon_{k}}\right\},\left\{\boldsymbol{v}^{\varepsilon_{k}}\right\},\left\{\boldsymbol{Z}^{\left(\tau_{k}\right)}\right\},\left\{\boldsymbol{U}^{\left(\tau_{k}\right)}\right\}$ of $\left\{\boldsymbol{u}^{\varepsilon}\right\},\left\{\boldsymbol{v}^{\varepsilon}\right\},\left\{\boldsymbol{Z}^{(\tau)}\right\},\left\{\boldsymbol{U}^{(\tau)}\right\}$, respectively, and a weak solution $\boldsymbol{z}$ of (5.1) such that

$$
\begin{array}{ll}
\mu \boldsymbol{u}^{\varepsilon_{k}}+\boldsymbol{v}^{\varepsilon_{k}} \rightarrow \boldsymbol{z} & \text { strongly in } L^{2}(Q)^{M}, \text { a.e. in } Q, \\
& \text { and weakly in } L^{2}\left(0, T ; H^{1}(\Omega)\right)^{M} \text { and } H^{1}\left(0, T ; H^{1}(\Omega)^{*}\right)^{M}, \\
\boldsymbol{u}^{\varepsilon_{k}} \rightarrow \boldsymbol{\beta}(\boldsymbol{z}) & \text { weakly in } L^{2}\left(0, T ; H^{1}(\Omega)\right)^{M}, \\
\boldsymbol{Z}^{\left(\tau_{k}\right)} \rightarrow \boldsymbol{z} & \text { strongly in } L^{2}(Q)^{M}, \text { a.e. in } Q \text { and weakly in } L^{2}\left(0, T ; H^{1}(\Omega)\right)^{M}, \\
\boldsymbol{U}^{\left(\tau_{k}\right)} \rightarrow \boldsymbol{\beta}(\boldsymbol{z}) & \text { weakly in } L^{2}\left(0, T ; H^{1}(\Omega)\right)^{M}
\end{array}
$$

as $\varepsilon_{k}, \tau_{k} \rightarrow 0$.

One-dimensional numerical experiments are carried out in a finite domain $\Omega=(0, L)=(0,3)$ and a time interval $(0, T]=(0,10]$. We employ the finite-difference method to discretize (5.2) in space. The spatial mesh size is denoted by $h=L / N_{X}$, where $N_{X}+1$ is the number of mesh points. Let $Z_{i}^{j, n}$ be the numerical approximation of $z_{i}(j h, n \tau)$. For given $\left\{Z_{i}^{j, n-1}\right\}_{i=1, \ldots, M, j=0, \ldots, N_{X}}\left(n=1, \ldots, N_{T}\right)$, solve the following linear 


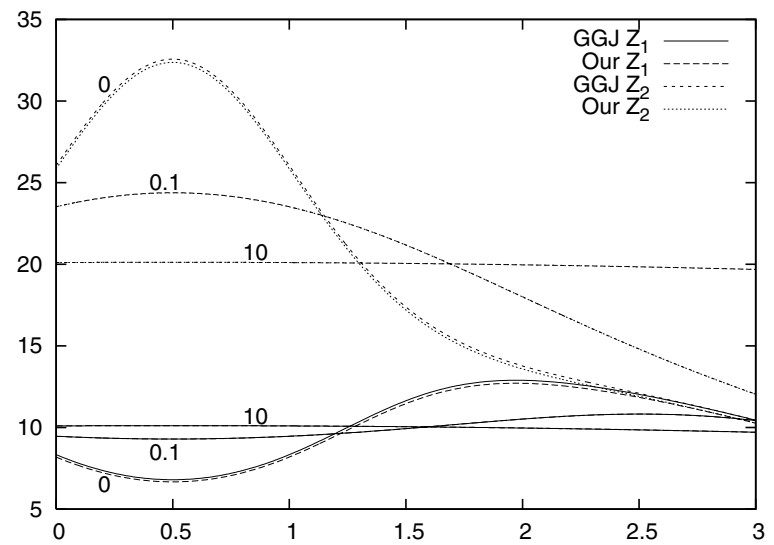

Figure 1. Numerical solutions of (5.1) corresponding to case A. Different curves are labelled with the corresponding $b_{i}$ values.

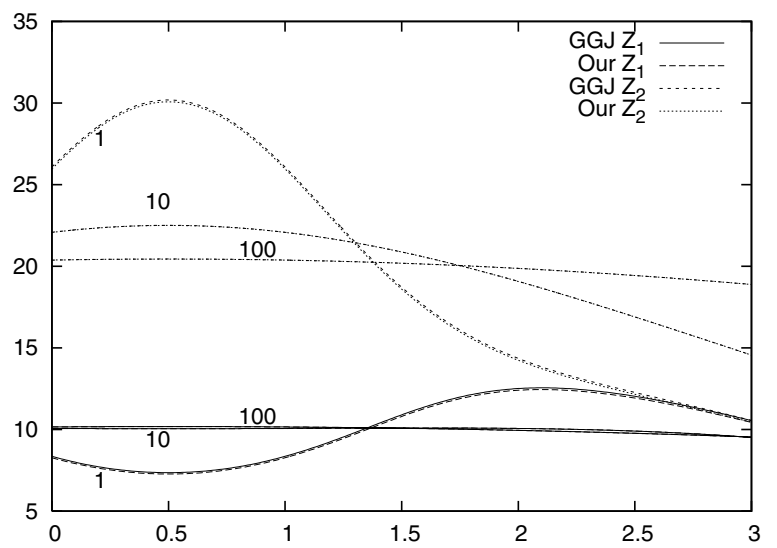

FIGURE 2. Numerical solutions of (5.1) corresponding to case B. Different curves are labelled with the corresponding $a_{i}$ values.

system to find $\left\{U_{i}^{j, n}\right\}_{i=1, \ldots, M, j=0, \ldots, N_{X}}$ :

$$
\begin{aligned}
& U_{i}^{j, n}-\frac{\tau}{\mu h^{2}}\left(U_{i}^{j+1, n}-2 U_{i}^{j, n}+U_{i}^{j-1, n}\right)-\frac{d_{i} \tau}{2 h}\left(U_{i}^{j+1, n}-U_{i}^{j-1, n}\right) p_{x}(j h)-d_{i} \tau U_{i}^{j, n} p_{x x}(j h) \\
& =\beta_{i}\left(\boldsymbol{Z}^{j, n-1}\right)+\frac{d_{i} \tau}{2 \mu h}\left(Z_{i}^{j+1, n-1}-Z_{i}^{j-1, n-1}-\mu \beta_{i}\left(\boldsymbol{Z}^{j+1, n-1}\right)+\mu \beta_{i}\left(\boldsymbol{Z}^{j-1, n-1}\right)\right) p_{x}(j h) \\
& \quad+\frac{d_{i} \tau}{\mu}\left(Z_{i}^{j, n-1}-\mu \beta_{i}\left(\boldsymbol{Z}^{j, n-1}\right)\right) p_{x x}(j h), \\
& \quad \frac{U_{i}^{-1, n}-U_{i}^{1, n}}{2 h}-d_{i}\left(Z_{i}^{0, n-1}-\mu\left(U_{i}^{0, n}-\beta_{i}\left(\boldsymbol{Z}^{0, n-1}\right)\right)\right) p_{x}(0)=0, \\
& \frac{U_{i}^{N_{X}+1, n}-U_{i}^{N_{X}-1, n}}{2 h}+d_{i}\left(Z_{i}^{N_{X}, n-1}-\mu\left(U_{i}^{N_{X}, n}-\beta_{i}\left(\boldsymbol{Z}^{N_{X}, n-1}\right)\right)\right) p_{x}(L)=0 .
\end{aligned}
$$

Thereafter, compute $\left\{Z_{i}^{j, n}\right\}_{i=1, \ldots, M, j=0, \ldots, N_{X}}$ by

$$
Z_{i}^{j, n}=Z_{i}^{j, n-1}+\mu\left(U_{i}^{j, n}-\beta_{i}\left(\boldsymbol{Z}^{j, n-1}\right)\right) .
$$

We repeat the experiments by Galiano et al. [7]. In each experiment, we set $M=2, \beta_{i}(\boldsymbol{z})=\left(a_{i}+b_{i} z_{i}+c_{i} z_{j}\right) z_{i}$ for $(i, j) \in\{(1,2),(2,1)\}, p(x)=1.5(x-0.5)^{2}, Z_{1}^{\cdot}, 0 \equiv 10, Z_{2}^{\cdot, 0} \equiv 20$. We take $h=10^{-2}, \tau=10^{-3}$ and $\mu=10^{-3}$. Numerical solutions by our method (denoted by Our) are compared with those by the method by Galiano et al. [7] (denoted by GGJ). We introduced the mind of GGJ in Section 1, but see [7] for the details.

We carry out numerical simulations in the following cases:

A: Large and small cross-diffusion terms compared to self-diffusion terms. The coefficients $a_{i}=c_{i}=d_{i}=1$ are fixed. Figure 1 shows the numerical solutions corresponding to $b_{i}=0,0.1,10$.

B: Large diffusion coefficients $a_{i}$ compared to $b_{i}$. The coefficients $b_{i}=0.01, c_{i}=d_{i}=1$ are fixed. Figure 2 shows the numerical solutions corresponding to $a_{i}=1,10,100$.

The numerical solutions by our method and by GGJ agree well (see also [1]). Figures 3 and 4 show close up view of Figure 1 near $(x, z)=(2,12.8)$ and $(x, z)=(0.5,32.5)$, respectively. A fine grid solution with $h=10^{-3}$ and $\tau=10^{-4}$ by GGJ is also drawn in order to compare our method with GGJ in the accuracy. Our numerical solution shows excellent agreement with the fine grid solution. We carried out the experiments in 


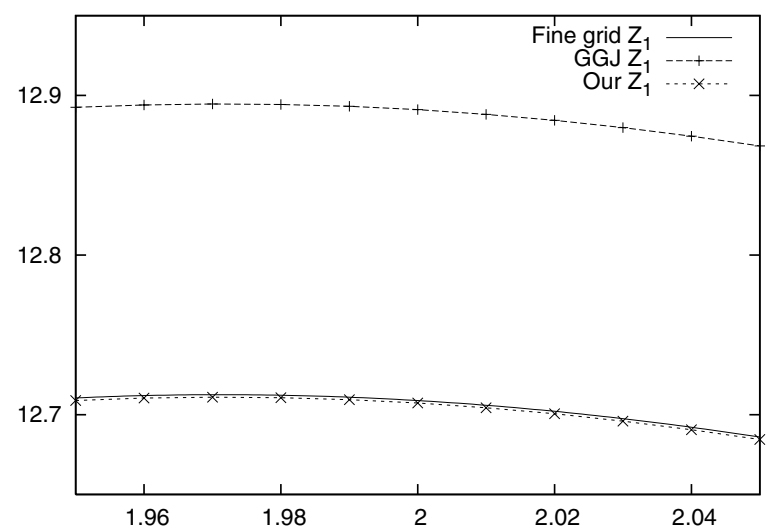

Figure 3. Close-up view of Figure 1 with a fine grid solution.

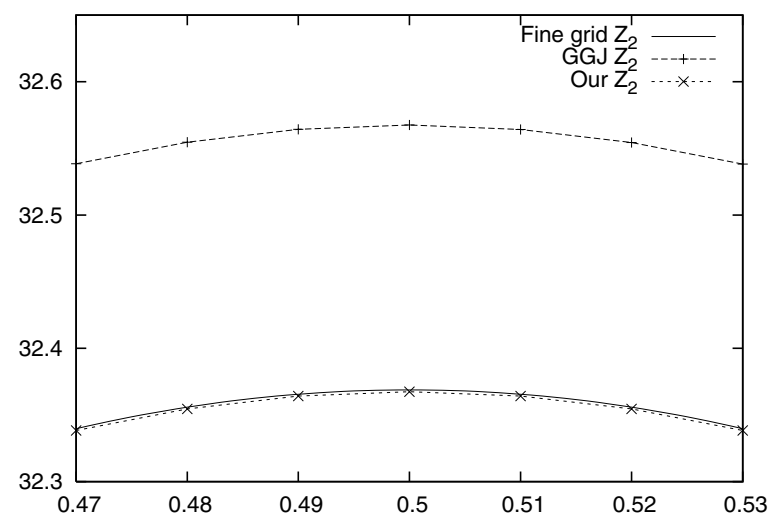

Figure 4. Close-up view of Figure 1 with a fine grid solution.

other cases treated in $[1,7]$. In all of those results, we observed that our numerical solutions agree well with fine grid solutions by GGJ. The numerical evidence demonstrates the effectiveness of our method. Furthermore, our method requires less CPU time than GGJ because the matrix dose not change in our method, i.e., we can take advantage of the LU decomposition to solve the linear systems.

\subsection{D simulations}

The advantages of our method are its simplicity and versatility. We can easily carry out two and three dimensional simulations for various type of nonlinearities. We present two dimensional simulations. In each simulation, we take the mesh sizes $h_{x}=h_{y}=1 / 500$, the time step size $\tau=10^{-4}$ and the parameter $\mu=0.2$. The following problem is considered.

$$
\begin{cases}\frac{\partial z_{1}}{\partial t}=\Delta\left[\left(0.04+0.04 \alpha z_{2}\right) z_{1}\right]+\left(2.8-1.1 z_{1}-z_{2}\right) z_{1} & \text { in } \quad Q=(0,1)^{2} \times(0,200] \\ \frac{\partial z_{2}}{\partial t}=\Delta\left[\left(0.04+2 \alpha z_{1}\right) z_{2}\right]+\left(3.0-1.1 z_{2}-z_{1}\right) z_{2} & \text { in } \quad Q\end{cases}
$$

with the homogeneous Neumann boundary conditions. If the cross-diffusion terms are not, that is, $\alpha=0$, the constant steady state $\left(z_{1}, z_{2}\right) \equiv(8 / 21,50 / 21)$ is stable. The stationary solution perturbed with $1 \%$ random noise is used for the initial datum. Figure 5 presents the numerical result with $\alpha=1$. The gray and the transparent light gray surfaces denote the numerical solutions $Z_{1}$ and $Z_{2}$, respectively. The stationary solution becomes unstable and a spatial pattern appears by the cross-diffusion effect.

We simulate the following cooperative system:

$$
\left\{\begin{array}{l}
\frac{\partial z_{1}}{\partial t}=\Delta\left[\left(\frac{1}{0.2+0.02 z_{2}^{2}}\right) z_{1}\right]+\left(4-z_{1}\right) z_{1} \quad \text { in } \quad Q=(0,1)^{2} \times(0,50], \\
\frac{\partial z_{2}}{\partial t}=0.005 \Delta z_{2}+\left(0.5+z_{1}-0.5 z_{2}\right) z_{2} \quad \text { in } \quad Q
\end{array}\right.
$$

with the homogeneous Neumann boundary conditions. The constant steady state $\left(z_{1}, z_{2}\right) \equiv(4,9)$ is stable in this problem if the cross-diffusion term is not. However, the stationary solution becomes unstable and aggregation occurs by the cross-diffusion effect (Fig. 6). Thus, we can simulate such complicated phenomena very easily by using the proposed scheme. 


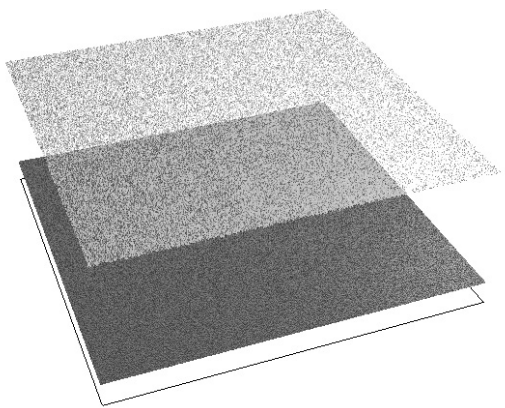

$t=0$.

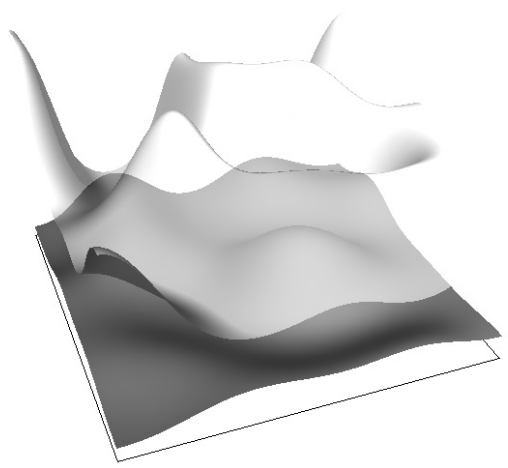

$t=25$.

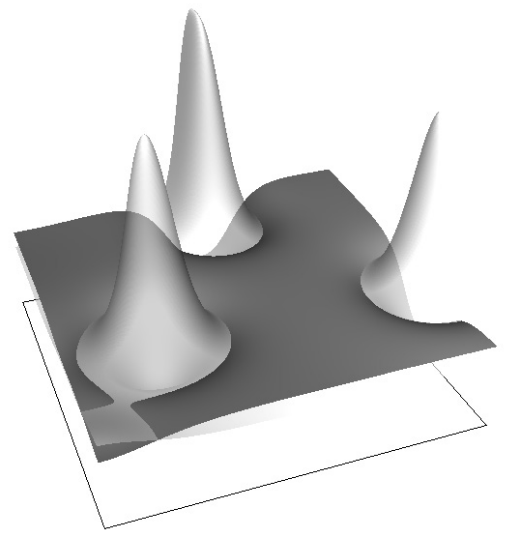

$t=40$.

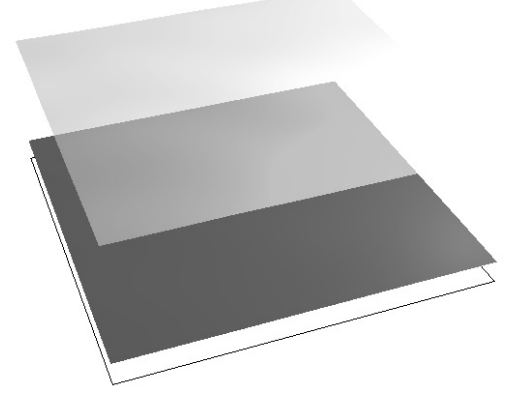

$t=15$.

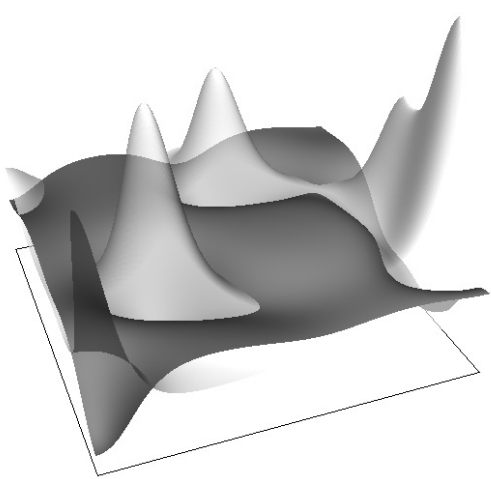

$t=30$

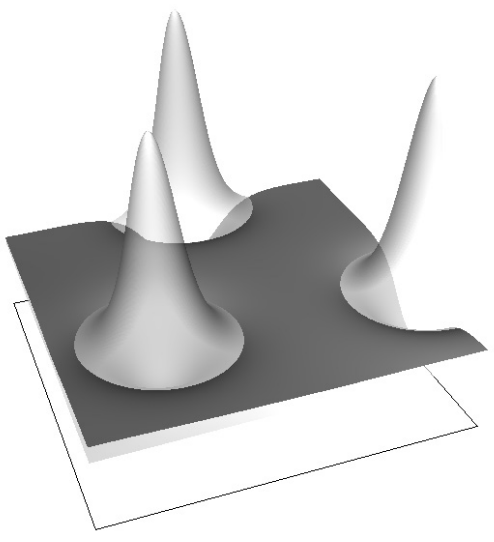

$t=100$.
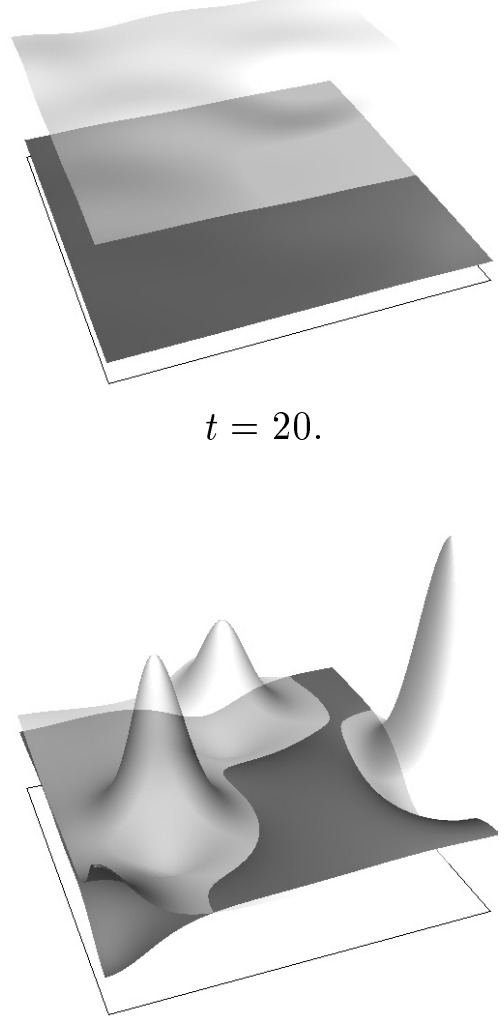

$t=35$.

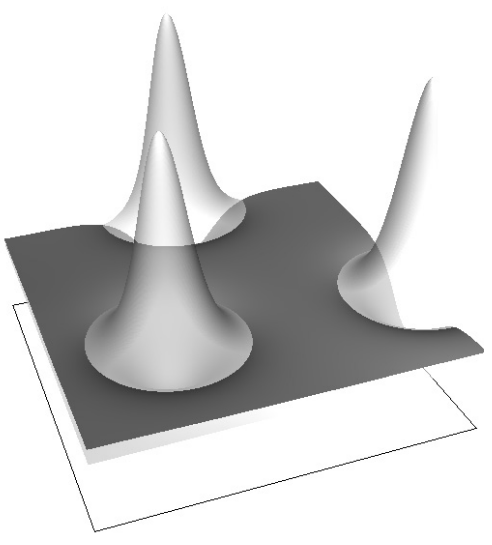

$t=200$.

FiguRE 5. Numerical solutions $Z_{1}$ (gray) and $Z_{2}$ (light gray) of (5.4). 

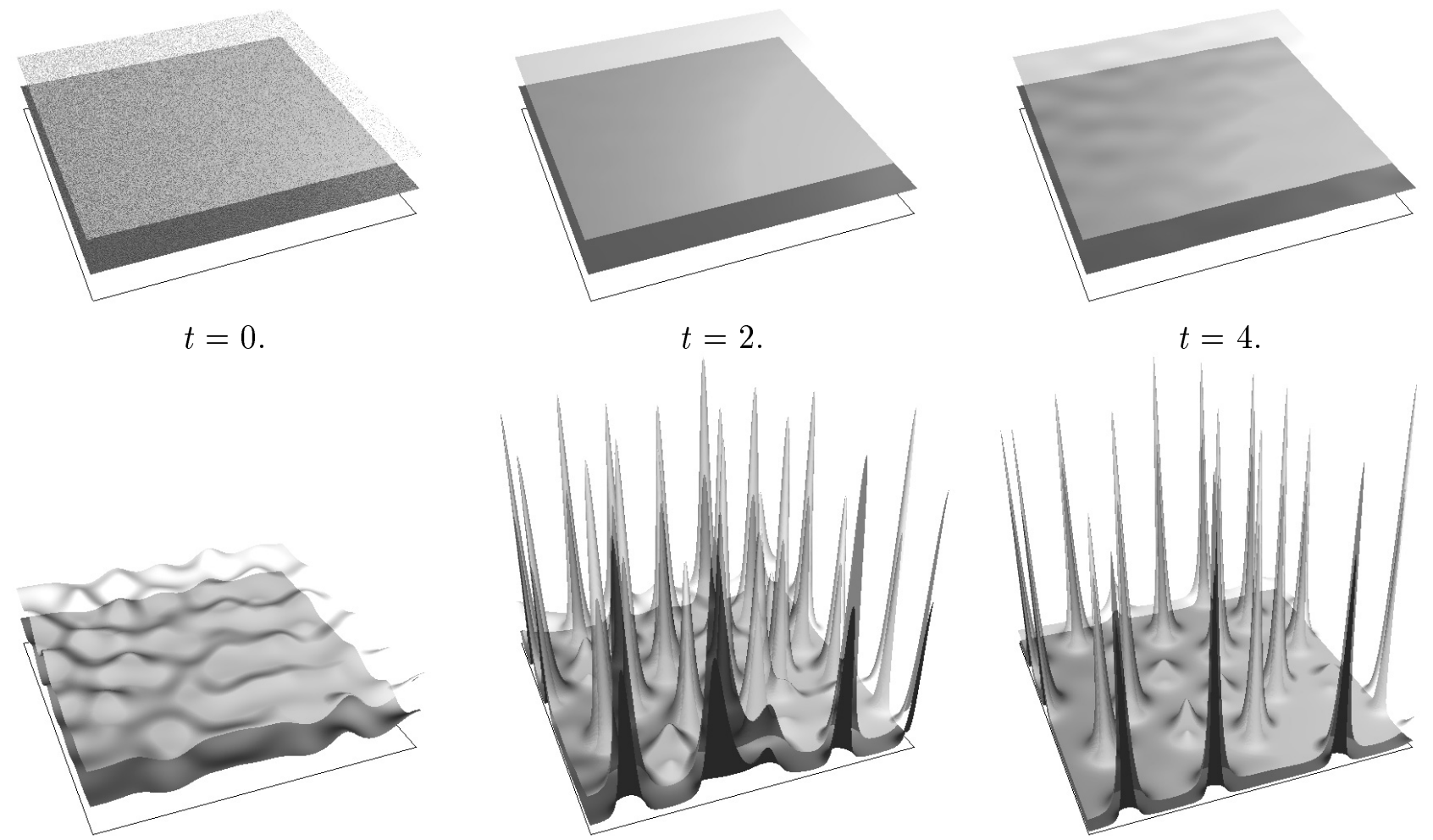

$t=6$.
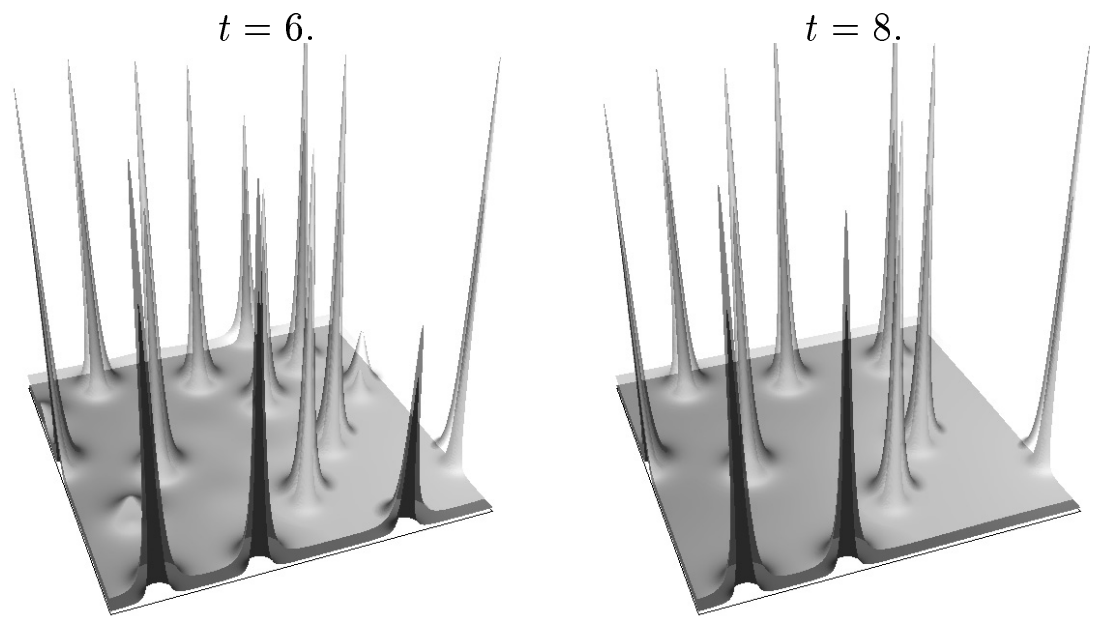

$t=12$.

$t=20$.

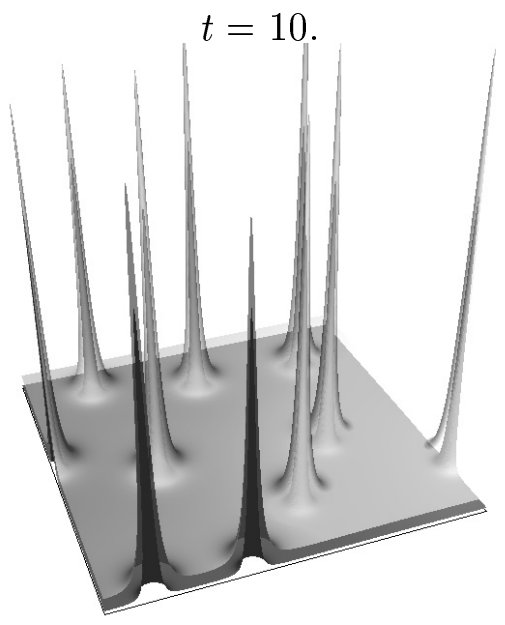

$t=50$.

FiguRE 6. Numerical solutions $Z_{1}$ (gray) and $Z_{2}$ (light gray) of (5.5). 


\section{REFERENCES}

[1] J.W. Barrett and J.F. Blowey, Finite element approximation of a nonlinear cross-diffusion population model. Numer. Math. 98 (2004) 195-221.

[2] G. Beckett, J.A. Mackenzie and M.L. Robertson, A moving mesh finite element method for the solution of two-dimensional Stefan problems. J. Comp. Phys. 168 (2001) 500-518.

[3] A.E. Berger, H. Brezis and J.C.W. Rogers, A numerical method for solving the problem $u_{t}-\Delta f(u)=0$. RAIRO Anal. Numer. 13 (1979) 297-312.

[4] H. Brézis, Analyse Fonctionnelle. Masson (1983).

[5] L. Chen and A. Jüngel, Analysis of a multidimensional parabolic population model with strong cross-diffusion. SIAM J. Math. Anal. 36 (2004) 301-322.

[6] L. Chen and A. Jüngel, Analysis of a parabolic cross-diffusion population model without self-diffusion. J. Differ. Equ. 224 (2006) 39-59.

[7] G. Galiano, M.L. Garzón and A. Jüngel, Analysis and numerical solution of a nonlinear cross-diffusion system arising in population dynamics. Rev. R. Acad. Cien. Ser. A Mat. 95 (2001) 281-295.

[8] G. Galiano, M.L. Garzón and A. Jüngel, Semi-discretization in time and numerical convergence of solutions of a nonlinear cross-diffusion population model. Numer. Math. 93 (2003) 655-673.

[9] M.E. Gurtin, Some mathematical models for population dynamics that lead to segregation. Quart. Appl. Math. 32 (1974) 1-9.

[10] W. Jäger and J. Kačur, Solution of porous medium type systems by linear approximation schemes. Numer. Math. 60 (1991) 407-427.

[11] J. Kačur, A. Handlovičová and M. Kačurová, Solution of nonlinear diffusion problems by linear approximation schemes. SIAM J. Numer. Anal. 30 (1993) 1703-1722.

[12] T. Kadota and K. Kuto, Positive steady states for a prey-predator model with some nonlinear diffusion terms. J. Math. Anal. Appl. 323 (2006) 1387-1401.

[13] E.H. Kerner, Further considerations on the statistical mechanics of biological associations. Bull. Math. Biophys. 21 (1959) $217-255$.

[14] E. Magenes, R.H. Nochetto and C. Verdi, Energy error estimates for a linear scheme to approximate nonlinear parabolic problems. Math. Mod. Numer. Anal. 21 (1987) 655-678.

[15] M. Mimura and K. Kawasaki, Spatial segregation in competitive interaction-diffusion equations. J. Math. Biol. 9 (1980) $49-64$.

[16] H. Murakawa, Reaction-diffusion system approximation to degenerate parabolic systems. Nonlinearity 20 (2007) $2319-2332$.

[17] H. Murakawa, A relation between cross-diffusion and reaction-diffusion. Discrete Contin. Dyn. Syst. S 5 (2012) $147-158$.

[18] R.H. Nochetto and C. Verdi, An efficient linear scheme to approximate parabolic free boundary problems: error estimates and implementation. Math. Comput. 51 (1988) 27-53.

[19] R.H. Nochetto and C. Verdi, The combined use of a nonlinear Chernoff formula with a regularization procedure for two-phase Stefan problems. Numer. Funct. Anal. Optim. 9 (1988) 1177-1192.

[20] R.H. Nochetto, M. Paolini and C. Verdi, An adaptive finite element method for two-phase Stefan problems in two space dimensions. Part I: stability and error estimates. Math. Comput. 57 (1991) 73-108.

[21] R.H. Nochetto, M. Paolini and C. Verdi, A fully discrete adaptive nonlinear Chernoff formula. SIAM J. Numer. Anal. 30 (1993) 991-1014.

[22] R.H. Nochetto, A. Schmidt and C. Verdi, A posteriori error estimation and adaptivity for degenerate parabolic problems. Math. Comput. 69 (1999) 1-24.

[23] P.Y.H. Pang and M.X. Wang, Strategy and stationary pattern in a three-species predator-prey model. J. Differ. Equ. 200 (2004) 245-273.

[24] N. Shigesada, K. Kawasaki and E. Teramoto, Spatial segregation of interacting species. J. Theor. Biol. 79 (1979) 83-99.

[25] R. Temam, Navier-Stokes equation theory and numerical analysis. AMS Chelsea Publishing, Providence, RI (2001).

[26] C. Verdi, Numerical aspects of parabolic free boundary and hysteresis problems. Lecture Notes in Mathematics 1584 (1994) 213-284. 